\title{
On the Fourier transform of regularized Bessel functions on complex numbers and Beyond Endoscopy over number fields
}

\author{
Zhi Qi
}

\begin{abstract}
Aвstract. In this article, we prove certain Weber-Schafheitlin type integral formulae for Bessel functions over complex numbers. A special case is a formula for the Fourier transform of regularized Bessel functions on complex numbers. This is applied to extend the work of A. Venkatesh on Beyond Endoscopy for $\mathrm{Sym}^{2}$ on $\mathrm{GL}_{2}$ from totally real to arbitrary number fields.
\end{abstract}

\section{Introduction}

A special case of the discontinuous integrals of Weber and Schafheitlin on the Fourier transform of Bessel functions on $\mathbb{R}_{+}=(0, \infty)$ is as follows (see [Wat 13.42 (2), (3)]),

$$
\int_{0}^{\infty} \frac{J_{v}(4 \pi x) e( \pm x y)}{x} d x= \begin{cases}\frac{2^{v}}{v\left(\sqrt{4-y^{2}} \mp i y\right)^{v}}, & \text { if } 0 \leqslant y \leqslant 2, \\ \frac{2^{v} e^{ \pm \frac{1}{2} \pi i v}}{v\left(\sqrt{y^{2}-4}+y\right)^{v}}, & \text { if } y>2,\end{cases}
$$

valid for $\operatorname{Re} v>0$, where $J_{v}(z)$ is the Bessel function of the first kind of order $v$ and as usual $e(z)=e^{2 \pi i z}$; it is assumed here that $\left.\arg \left(\sqrt{4-y^{2}} \mp i y\right) \in \mp\left[0, \frac{1}{2} \pi\right] 1\right]$ Subsequently, we shall call a formula of this kind special Weber-Schafheitlin integral formula.

In Venkatesh's work on Beyond Endoscopy [Ven2], the second formula in 1.11 arises in his local computation over $\mathbb{R}$, particularly, in his analysis for the $B$-transform. The results of Venkatesh were proven for totally real number fields, but he pointed out that the extension to complex places would only require verifying a similar formula for Bessel functions over $\mathbb{C}$. This amounts to a "local fundamental lemma" over $\mathbb{C}$. Unfortunately, it seems to resist a proof in every direct way-Venkatesh was not able to prove it at that time.

The purpose of this paper is to establish an integral formula for Bessel functions over complex numbers which is analogous to the special Weber-Schafheitlin formula as in (1.1) (after regularization). Our approach is a rather indirect method that combines asymptotic

2010 Mathematics Subject Classification. 33C10, 42B10, 33C05.

Key words and phrases. Fourier transform, Bessel functions, hypergeometric function, Beyond Endoscopy. ${ }^{1}$ The first line on the right hand side of 1.1 should read $(1 / v) \exp ( \pm i v \arcsin (y / 2))$ by bookkeeping Wat 13.42 (2), (3)], but we feel that the formulation here in 1.1 is more suggestive. 
analysis and differential equations. As an application, the validity of Venkatesh's work on Beyond Endoscopy is extended from totally real to arbitrary number fields.

\subsection{Analysis over real numbers.}

Bessel kernels and the special Weber-Schafheitlin formula. First of all, some remarks on the special Weber-Schafheitlin formula (1.1) are in order, as the motivation of our investigation of its complex analogue.

The Bessel function of concern has pure imaginary order $v= \pm 2 i t$ ( $t$ real). Indeed, the Bessel kernel in the $B$-transform is $B_{2 i t}(4 \pi x)$ (see [Ven2, §6.7.2]), with

$$
B_{v}(x)=\frac{1}{\sin (\pi v / 2)}\left(J_{-v}(x)-J_{v}(x)\right), \quad x \in \mathbb{R}_{+},
$$

and the $B$-transform is defined by

$$
\phi(x)=\frac{1}{2} \int_{-\infty}^{\infty} B_{2 i t}(4 \pi x) h(t) \sinh (\pi t) t d t .
$$

The formula (1.1) however is only valid for $\operatorname{Re} v>0$. The reason is that $J_{v}(4 \pi x) \sim$ $(2 \pi x)^{v} / \Gamma(v+1)$ as $x \rightarrow 0$ (see [Wat $\left.3.1(8)\right]$ ), say for $\operatorname{Re} v \geqslant 0$, and hence the integral in (1.1) is convergent near zero only when $\operatorname{Re} v>0$. It should be noted that the integral is always absolutely convergent in the vicinity of infinity since $J_{v}(4 \pi x)=O(1 / \sqrt{x})$ as $x \rightarrow \infty$ (see [Wat 7.21 (1)]). Nevertheless, this convergence issue may be easily addressed by shifting $t$ to $t-i \sigma$ or $t+i \sigma(\sigma>0)$ for $J_{2 i t}(4 \pi x)$ or $J_{-2 i t}(4 \pi x)$ respectively.

The Bessel kernel in the $K$-transform is $(4 / \pi) \cos (\pi t) K_{2 i t}(4 \pi x)$ (see [Ven2, §6.7.3]), with

$$
K_{v}(x)=\frac{\pi}{2 \sin (\pi v)}\left(I_{-v}(x)-I_{v}(x)\right), \quad x \in \mathbb{R}_{+},
$$

where $I_{v}(z)$ and $K_{v}(z)$ are the modified Bessel functions 2 . The $K$-transform is defined by

$$
\phi(x)=\frac{1}{\pi} \int_{-\infty}^{\infty} K_{2 i t}(4 \pi x) h(t) \sinh (2 \pi t) t d t .
$$

The integral in 1.1 would diverge if the $J_{v}(4 \pi x)$ were replaced by $I_{v}(4 \pi x)$ since it is of exponential growth $\left(I_{v}(x) \sim \exp (x) / \sqrt{2 \pi x}\right.$, [Wat 7.23 (2), (3)]) as $x \rightarrow \infty$. Although $I_{v}(x)$ and $I_{-v}(x)$ conspire in (1.4) so that $K_{v}(x)$ decays exponentially $\left(K_{v}(x) \sim\right.$ $\exp (-x) / \sqrt{2 x / \pi}$, [Wat $7.23(1)])$ as $x \rightarrow \infty$, the integral in 1.1) would still diverge if the $J_{v}(4 \pi x)$ were replaced by $K_{v}(4 \pi x)$. This is because $I_{v}(4 \pi x) \sim(2 \pi x)^{v} / \Gamma(v+1)$ as $x \rightarrow 0$ (see [Wat 3.7 (2)]) and it would require both $\operatorname{Re} v>0$ and $\operatorname{Re}(-v)>0$ for the integral to be convergent near zero, which is clearly impossible. At any rate, the special WeberSchafheitlin formula does not exist for the Bessel kernel of the $K$-transform. Moreover, in the case $v=2 i t$, the order-shifting trick as above applied to $I_{2 i t}(4 \pi x)$ and $I_{-2 i t}(4 \pi x)$ would not work: after the order-shifting their exponential growth could not be canceled completely and the resulting difference $I_{-2 i t+2 \sigma}(4 \pi x)-I_{2 i t+2 \sigma}(4 \pi x)(\sigma>0)$ still grows exponentially (compare their asymptotic expansions as in [Wat 7.23 (2), (3)]). As such,

\footnotetext{
${ }^{2}$ Note that the $\sin (\pi v)$ is mistakenly written $\operatorname{as} \sin (\pi v / 2)$ in Ven2.
} 
the analysis for the $K$-transform in [Ven2, §6.7.3] is quite different from that for the $B$ transform in [Ven2, §6.7.2]. Instead, Venkatesh uses the Lebedev-Kontorovitch inversion and the Mehler-Sonine integral representation for $K_{2 i t}(x)$.

Similar obstacles described above for $K_{2 i t}(4 \pi x)$ would arise when analyzing the Fourier transform of Bessel functions on $\mathbb{C}$. Thus before we initiate our study over $\mathbb{C}$ it is necessary to overcome these obstacles over $\mathbb{R}$ with new ideas.

Regularizing the special Weber-Schafheitlin formula. Next, we introduce the regularized Bessel kernels and their special Weber-Schafheitlin formulae and briefly explain how they are applied to unify the analysis for the $B$-transform and that for the $K$-transform. The merit of unified analyses for the $B$ - and the $K$-transform and also for their Bessel kernels is that their complex extensions are usually admissible.

We first define

$$
P_{v}(x)=\frac{(x / 2)^{v}}{\Gamma(v+1)}
$$

Note that $P_{v}(x)$ is simply the leading term in the series expansion of $J_{v}(x)$ or $I_{v}(x)$ at $x=0$ (see [Wat 3.1 (8), 3.7 (2)]). By [GR, 3.764], we have

$$
\int_{0}^{\infty} x^{\nu-1} e( \pm x y) d x=\Gamma(v) e^{ \pm \frac{1}{2} \pi i v}(2 \pi y)^{-v}, \quad 0<\operatorname{Re} v<1 .
$$

In the following, let $\operatorname{Re} v=0$ and, for simplicity, $v \neq 0$. The results may be extended to $v=0$ and even to all $v$ with $|\operatorname{Re} v|<1$ by the principle of analytic continuation.

We now define

$$
R_{v}(x)=\frac{1}{\sin (\pi v / 2)}\left(P_{-v}(x)-P_{v}(x)\right)
$$

and the regularized Bessel kernels

$$
D_{v}(x)=B_{v}(x)-R_{v}(x), \quad M_{v}(x)=(4 / \pi) \cos (\pi v / 2) K_{v}(x)-R_{v}(x) .
$$

Consider the following two integrals

$$
\int_{0}^{\infty} \frac{D_{v}(4 \pi x) e( \pm x y)}{x} d x, \quad \int_{0}^{\infty} \frac{M_{v}(4 \pi x) e( \pm x y)}{x} d x .
$$

We have $J_{v}(x)-P_{v}(x), I_{v}(x)-P_{v}(x)=O\left(x^{2}\right)$ as $x \rightarrow 0$. Thus these integrals are absolutely convergent at zero and become so after integration by parts in the vicinity of infinity (the region of convergence may actually be extended to $|\operatorname{Re} v|<1$ and the convergence is uniform for $\operatorname{Re} v$ in a compact set).

For the first integral in (1.10), we apply (1.1) and (1.7) to the Fourier transform of $\left(J_{-v+\sigma}(4 \pi x)-P_{-v+\sigma}(4 \pi x)\right) / x$ and $\left(J_{v+\sigma}(4 \pi x)-P_{\nu+\sigma}(4 \pi x)\right) / x$ and then let $\sigma \rightarrow 0$; the uniformity in $\operatorname{Re} \sigma$ may be easily verified. In this way, we obtain for $y>2$ that

$$
\begin{aligned}
\int_{0}^{\infty} \frac{D_{\nu}(4 \pi x) e( \pm x y)}{x} d x=-\frac{1}{v \sin (\pi v / 2)}\left\{e^{\mp \frac{1}{2} \pi i v}\left(\frac{\left(y+\sqrt{y^{2}-4}\right)^{v}}{2^{v}}-y^{v}\right)\right. \\
\left.+e^{ \pm \frac{1}{2} \pi i v}\left(\frac{2^{v}}{\left(y+\sqrt{y^{2}-4}\right)^{v}}-\frac{1}{y^{v}}\right)\right\} .
\end{aligned}
$$

The formula in the case $0 \leqslant y \leqslant 2$ is similar. 
For the second integral in (1.10), as indicated before, there is no special WeberSchafheitlin formula for either $I_{v}(4 \pi x)$ or $K_{v}(4 \pi x)$. We propose an alternative approach by modifying the integrals by the factor $x^{\rho}$ with $0<\operatorname{Re} \rho<1$. By [GR 6.699 3, 4] and the transformation formula for hypergeometric functions with respect to $z \rightarrow 1 / z$ (see [MOS] §2.4.1]), along with the reflection and the duplication formula for the gamma function, we infer that for all $\operatorname{Re} \rho>0$

$$
\begin{aligned}
\int_{0}^{\infty} K_{\nu}(4 \pi x) e( \pm x y) x^{\rho-1} d x= & \\
\frac{\pi}{2(2 \pi)^{\rho} \sin (\pi v)} & \left\{\frac{\Gamma(\rho-v) e^{ \pm \frac{1}{2} \pi i(\rho-v)}}{\Gamma(1-v) y^{\rho-v}}{ }_{2} F_{1}\left(\frac{\rho-v}{2}, \frac{1+\rho-v}{2} ; 1-v ;-\frac{4}{y^{2}}\right)\right. \\
& \left.-\frac{\Gamma(\rho+v) e^{ \pm \frac{1}{2} \pi(\rho+v)}}{\Gamma(1+v) y^{\rho+v}}{ }_{2} F_{1}\left(\frac{\rho+v}{2}, \frac{1+\rho+v}{2} ; 1+v ;-\frac{4}{y^{2}}\right)\right\} .
\end{aligned}
$$

A formula of this kind will be called general Weber-Schafheitlin integral formula. We now apply (1.7) and (1.12) to the Fourier transform of $(4 / \pi) \cos (\pi v / 2) K_{v}(4 \pi x) x^{\rho-1}-$ $\left(P_{\rho-\nu}(4 \pi x)-P_{\rho+\nu}(4 \pi x)\right) / \sin (\pi v / 2)$ and then let $\rho \rightarrow 0$. The resulting hypergeometric functions may be evaluated by the formula (see [MOS §2.1])

$$
\left(1+\sqrt{1-z^{2}}\right)^{-2 a}=2^{-2 a}{ }_{2} F_{1}\left(a, a+1 / 2 ; 2 a+1 ; z^{2}\right) .
$$

Finally we obtain

$$
\begin{aligned}
\int_{0}^{\infty} \frac{M_{v}(4 \pi x) e( \pm x y)}{x} d x=-\frac{1}{v \sin (\pi v / 2)} & \left\{e^{\mp \frac{1}{2} \pi i v}\left(\frac{\left(y+\sqrt{y^{2}+4}\right)^{v}}{2^{v}}-y^{v}\right)\right. \\
+e^{ \pm \frac{1}{2} \pi i v} & \left.\left(\frac{2^{v}}{\left(y+\sqrt{y^{2}+4}\right)^{v}}-\frac{1}{y^{v}}\right)\right\},
\end{aligned}
$$

which is very similar to (1.11). This somewhat indirect approach to 1.14 would also lead us to 1.11); we only record here the general Weber-Schafheitlin integral formula for $J_{v}(4 \pi x)$ as below in the case $y>2($ see $[\mathbf{G R}, 6.6991,2])$,

$$
\int_{0}^{\infty} J_{v}(4 \pi x) e( \pm x y) x^{\rho-1} d x=\frac{\Gamma(\rho+v) e^{ \pm \frac{1}{2} \pi i(\rho+v)}}{(2 \pi)^{\rho} \Gamma(1+v) y^{\rho+v}}{ }_{2} F_{1}\left(\frac{\rho+v}{2}, \frac{1+\rho+v}{2} ; 1+v ; \frac{4}{y^{2}}\right)
$$

valid for $0<\operatorname{Re} \rho<\frac{3}{2}$.

An observation is that the paired functions in (1.11) and (1.14), say $\left(y+\sqrt{y^{2}-4}\right)^{v} / 2^{v}$ and $y^{v}$, are asymptotically equivalent as $y \rightarrow \infty$. In other words, the Fourier transform of $B_{v}(4 \pi x) / x$ or $4 \cos (\pi v / 2) K_{v}(4 \pi x) / \pi x$ and that of $R_{v}(4 \pi x) / x$, in the formal sense, have the same asymptotic at infinity. Note that this is also true if they are modified by the factor $x^{\rho}$. A similar observation, Lemma 4.2 is crucial to our analysis over complex numbers. See $\$ 4.1$ for more details. 
For the analysis for the $B$-transform in [Ven2, §6.7.2], we modify the arguments therein as follows. First, we start with writing

$$
\begin{aligned}
\int_{0}^{\infty} \cos (2 \pi k x) \phi(x) \frac{d x}{x}= & \frac{1}{2} \int_{0}^{\infty} \cos (2 \pi k x) \int_{-\infty}^{\infty} D_{2 i t}(4 \pi x) h(t) \sinh (\pi t) t d t \frac{d x}{x} \\
& +i \int_{0}^{\infty} \cos (2 \pi k x) \int_{-\infty}^{\infty} P_{2 i t}(4 \pi x) h(t) t d t \frac{d x}{x},
\end{aligned}
$$

instead of

$$
\int_{0}^{\infty} \cos (2 \pi k x) \phi(x) \frac{d x}{x}=i \int_{0}^{\infty} \cos (2 \pi k x) \int_{-\infty}^{\infty} J_{2 i t}(4 \pi x) h(t) t d t \frac{d x}{x} .
$$

Second, we apply (1.11) directly to the first integral on the right of (1.16), and shift the order of $P_{2 i t}(4 \pi x)$ in (1.16) instead of that of $J_{2 i t}(4 \pi x)$ in (1.17). Note that the $B$-transform turns into the inverse Mellin transform if $J_{2 i t}(4 \pi x)$ is substituted by $P_{2 i t}(4 \pi x)$. It is clear that the analysis for the $K$-transform may also be done in this way. Since these will be elaborated in the complex setting in $\$ 6$ we do not discuss here any further details.

The ideas outlined above will be executed in $\S \S 5$ and 6 for our analysis on complex numbers. Yet we still need to first establish the complex analogue of the general WeberSchafheitlin integral formula in (1.12) or (1.15). This is actually the main technical result of this article; its proof will be given mainly in $\$ 4$

1.2. Main theorems. We now introduce the definition of Bessel functions over complex numbers (see [Qi1 §15.3], [BM5, (6.21), (7.21)]). Let $\mu$ be a complex number and $d$ be an integer. We define

$$
J_{\mu, d}(z)=J_{\mu+d}(z) J_{\mu-d}(\bar{z}) .
$$

The function $J_{\mu, d}(z)$ is well defined and even on $\mathbb{C} \backslash\{0\}$ in the sense that the expression on the right of $(1.18)$ is independent on the choice of the argument of $z$ modulo $\pi$. Next, we define

$$
\boldsymbol{J}_{\mu, d}(z)=\frac{1}{\sin (\pi \mu)}\left(J_{-\mu,-d}(4 \pi z)-J_{\mu, d}(4 \pi z)\right) .
$$

It is understood that in the non-generic case when $\mu$ is an integer the right hand side should be replaced by its limit. It is clear that $\boldsymbol{J}_{\mu, d}(z)$ is also an even function on $\mathbb{C} \backslash\{0\}$.

According to [Qi1, §18.2], these Bessel functions are attached to representations of $\mathrm{PGL}_{2}(\mathbb{C})$. We shall not restrict ourselves to the Bessel functions of trivial $\mathrm{SU}_{2}$-type $(d=$ 0 ) arising in the Kuznetsov-Bruggeman-Miatello formula (see [Ven2, §2.6, Appendix] or $[\text { BM4] }]^{3}$. For Bessel functions for $\mathrm{GL}_{2}(\mathbb{C})$ with non-trivial central characters, our results and method would still be valid but the formulae would be more involved. For these we refer the reader to Appendix $\mathrm{A}$

General Weber-Schafheitlin formula. First, we have the general Weber-Schafheitlin integral formula for $\boldsymbol{J}_{\mu, d}(z)$ as follows.

THeorem 1.1. Suppose that $|\operatorname{Re} \mu|<\operatorname{Re} \rho<\frac{1}{2}$. Define

$$
C_{\mu, d}^{\rho}=-\frac{\sin (\pi(\rho+\mu))}{(2 \pi)^{2 \rho} \sin (\pi \mu)} \frac{\Gamma(\rho+\mu+d) \Gamma(\rho+\mu-d)}{\Gamma(1+\mu+d) \Gamma(1+\mu-d)},
$$

\footnotetext{
${ }^{3}$ For succinctness, we shall suppress $d$ from our notation if $d=0$, so in particular $\boldsymbol{J}_{\mu}(z)=\boldsymbol{J}_{\mu, 0}(z)$.
} 
and

(1.21)

$F_{\rho, v}^{(1)}(z)={ }_{2} F_{1}\left(\frac{\rho+v}{2} ; \frac{1+\rho+v}{2} ; 1+v ; z\right), F_{\rho, v}^{(2)}(z)={ }_{2} F_{1}\left(\frac{\rho-v}{2} ; \frac{1+\rho-v}{2} ; 1-v ; z\right)$.

We have the identity

$$
\begin{aligned}
& \int_{0}^{2 \pi} \int_{0}^{\infty} \boldsymbol{J}_{\mu, d}\left(x e^{i \phi}\right) e(-2 x y \cos (\phi+\theta)) x^{2 \rho-1} d x d \phi= \\
& \frac{C_{\mu, d}^{\rho} F_{\rho, \mu+d}^{(1)}\left(4 / y^{2} e^{2 i \theta}\right) F_{\rho, \mu-d}^{(1)}\left(4 e^{2 i \theta} / y^{2}\right)}{y^{2 \rho+2 \mu} e^{2 i d \theta}}+\frac{C_{-\mu,-d}^{\rho} F_{\rho, \mu+d}^{(2)}\left(4 / y^{2} e^{2 i \theta}\right) F_{\rho, \mu-d}^{(2)}\left(4 e^{2 i \theta} / y^{2}\right)}{y^{2 \rho-2 \mu} e^{-2 i d \theta}}
\end{aligned}
$$

for $y \in[0, \infty)$ and $\theta \in[0,2 \pi)$; for $d=0$, the right hand side of the identity is to be replaced by its limit if $\mu=0$. Moreover, the identity (1.22) is valid under the weaker condition $|\operatorname{Re} \mu|<\operatorname{Re} \rho<1$ if we further assume that $y>2$.

REMARK 1.2. Since $F_{\rho, v}^{(1)}(1 / z)$ and $F_{\rho, v}^{(2)}(1 / z)$ are defined via analytic continuation within the unit circle $|z|<1$, the formula 1.22) is not quite illuminating for $y<2$; it is not so clear a priori that the right hand side of (1.22) is a well-defined function on the complex plane. An alternative expression of (1.22) obtained from Proposition 3.2 would be more transparent in terms of the Gauss hypergeometric series for $y<2$.

By the Gaussian formula for ${ }_{2} F_{1}(a, b ; c ; 1)$ (see [MOS \$2.1])

$$
{ }_{2} F_{1}(a, b ; c ; 1)=\frac{\Gamma(c) \Gamma(c-a-b)}{\Gamma(c-a) \Gamma(c-b)}, \quad \operatorname{Re}(a+b-c)<0, c \neq 0,-1,-2, \ldots,
$$

together with the duplication and the reflection formula for the gamma function, it is straightforward to derive the following corollary.

Corollary 1.3. For $|\operatorname{Re} \mu|<\operatorname{Re} \rho<\frac{1}{2}$, we have

$$
\begin{aligned}
& \int_{0}^{2 \pi} \int_{0}^{\infty} \boldsymbol{J}_{\mu, d}\left(x e^{i \phi}\right) e(-4 x \cos \phi) x^{2 \rho-1} d x d \phi=\left(2 / \pi^{3}\right)(8 \pi)^{-2 \rho} \cos (\pi \rho) \Gamma(1 / 2-\rho)^{2} . \\
& \sin (\pi(\rho+\mu)) \sin (\pi(\rho-\mu)) \Gamma(\rho+\mu+d) \Gamma(\rho+\mu-d) \Gamma(\rho-\mu+d) \Gamma(\rho-\mu-d) .
\end{aligned}
$$

We shall prove Theorem 1.1 by exploiting a soft method that combines asymptotic analysis of oscillatory integrals and a uniqueness result for differential equations. Precisely, it will be shown that the two sides of (1.22) satisfy the same asymptotic as $y \rightarrow \infty$ and also the same (hypergeometric) differential equations and hence are forced to be equal.

This method of proof grew out of the author's previous work [Qi3] on a similarlooking integral which, in the notation of this paper, may be written as follows

$$
\int_{0}^{2 \pi} \int_{0}^{\infty} \boldsymbol{J}_{\mu, d}\left(x^{\frac{1}{2}} e^{\frac{1}{2} i \phi}\right) e(-2 x y \cos (\phi+\theta)) d x d \phi .
$$

This Fourier-transform integral is however entirely different in nature. Roughly speaking, it has an explicit formula in terms of the Bessel function of halved order $\boldsymbol{J}_{\frac{1}{2} \mu, \frac{1}{2} d}\left(1 / 4 y e^{i \theta}\right)$ (see [Qi3] for the formula (in slightly different notation)). That formula was used to extend the Waldspurger formula of Baruch and Mao from totally real to arbitrary number fields; see [BM1, BM2, BM3] and [CQ1, CQ2]. The $x^{\frac{1}{2}} e^{\frac{1}{2} i \phi}$ above in 1.23 is squared into 
the $x e^{i \phi}$ in (1.22) (and also in (1.28), since it is the symmetric square lift $\mathrm{Sym}^{2}$ under consideration.

Any direct method seems impossible as there is no formula available in the literature to deal with the radial integration - the most outstanding obstacle in the extension of integral formulae from real to complex numbers. It is extremely lucky that the formula for (1.23) can be proven by known formulae in the spherical case $(d=0)$; see [Qi2]. For the integral under consideration, however, even the spherical case is inaccessible by direct method.

Special Weber-Schafheitlin formula after regularization. Define

$$
P_{\mu, d}(z)=\frac{(z / 2)^{\mu+d}(\bar{z} / 2)^{\mu-d}}{\Gamma(\mu+d+1) \Gamma(\mu-d+1)},
$$

and

$$
\boldsymbol{R}_{\mu, d}(z)=\frac{1}{\sin (\pi \mu)}\left(P_{-\mu,-d}(4 \pi z)-P_{\mu, d}(4 \pi z)\right) .
$$

Again, the right hand side is replaced by its limit when $\mu$ is an integer. We now state the regularized special Weber-Schafheitlin integral formula for $\boldsymbol{J}_{\mu, d}(z)$ as follows.

THEOREM 1.4. Suppose that $|\operatorname{Re} \mu|<\frac{1}{2}$. Define the regularized Bessel function

$$
\boldsymbol{M}_{\mu, d}(z)=\boldsymbol{J}_{\mu, d}(z)-\boldsymbol{R}_{\mu, d}(z) .
$$

Let

$$
Y(z)=\frac{\left|z+\sqrt{z^{2}-4}\right|}{2}, \quad E(z)=\frac{z+\sqrt{z^{2}-4}}{\left|z+\sqrt{z^{2}-4}\right|} .
$$

We have

$$
\begin{aligned}
\int_{0}^{2 \pi} \int_{0}^{\infty} \boldsymbol{M}_{\mu, d}\left(x e^{i \phi}\right) e(-2 x y \cos (\phi+\theta)) \frac{d x d \phi}{x}=\frac{1}{d^{2}-\mu^{2}} \\
\left\{\left(Y\left(y e^{i \theta}\right)^{2 \mu} E\left(y e^{i \theta}\right)^{2 d}-y^{2 \mu} e^{2 i d \theta}\right)+\left(\frac{1}{Y\left(y e^{i \theta}\right)^{2 \mu} E\left(y e^{i \theta}\right)^{2 d}}-\frac{1}{y^{2 \mu} e^{2 i d \theta}}\right)\right\}
\end{aligned}
$$

for $y \in(0, \infty)$ and $\theta \in[0,2 \pi)$; for $d=0$, the right hand side of the identity is to be replaced by its limit if $\mu=0$.

1.3. Application to Beyond Endoscopy over arbitrary number fields. In the work of Venkatesh [Ven2], Langlands' proposal of Beyond Endoscopy [Lan] is executed for the symmetric square lift $\mathrm{Sym}^{2}$ on $\mathrm{GL}_{2}$, giving the classification of dihedral forms-forms whose symmetric square has a pole. It is proven that dihedral forms correspond to Grössencharacters of quadratic field extensions. This result is originally due to Labesse and Langlands $[\mathbf{L L}]$ by endoscopic methods.

The fundamental tool used by Venkatesh at the beginning is the Kuznetsov relative trace formula of Bruggeman and Miatello. Poisson summation is then applied at the stage after Kuznetsov-Bruggeman-Miatello. Afterwards, the local exponential sums are evaluated and units of the quadratic field extension enter into the analysis. The Archimedean theory of Venkatesh is contained in his Proposition 7, in which arises naturally the Fourier transform of Bessel functions due to Kuznetsov-Bruggeman-Miatello and Poisson. 
Venkatesh works over a number field. As such, the method is extremely notationally complicated in his $\S 4$. He however takes great care in guiding the reader by illustrating the main ideas over $\mathbb{Q}$ in his $§ 3$. More details may be found in his thesis [Ven1].

The main results of Venkatesh (in his §4) are stated in the setting of a totally real number field. The only serious obstacle in the general case (involving complex places) is the validity of a certain integral transform as in his Proposition 7. Except for this, his method clearly does not rely on the totally real assumption in any important way.

In the present paper, Proposition 7 of Venkatesh is extended to complex places so that his main results are generalized to arbitrary number fields.

For other works on Beyond Endoscopy, see for example [Sar, Her1, Her2, Her3, FLN, Sak1, Sak2, Whi, Alt1, Alt2, Alt3].

Notation. Let $F$ be an (arbitrary) number field. Let $v$ stand for a place of $F$ and $F_{v}$ denote the completion of $F$ at $v$. Let $S_{\infty}$ be the set of Archimedean places. Write $v \mid \infty$ as the abbreviation for $v \in S_{\infty}$. Define $F_{\infty}$ to be $F \otimes \mathbb{R}=\prod_{v \mid \infty} F_{v}$. For $x \in F_{\infty}$ let $\operatorname{Norm}(x)$ denote the norm of $x$. We fix the Haar measure on $F_{\infty}$ corresponding to the product of $d x$ at real places and $|d x \wedge d \bar{x}|$ at complex places. Let $\psi_{\infty}: F_{\infty} \rightarrow \mathbb{C}$ be the additive character $\psi_{\infty}(x)=e\left(\operatorname{Tr}_{F_{\infty} / \mathbb{R}}(x)\right)$.

Let $\mathfrak{a}$ be the vector space $\mathbb{R}^{\left|S_{\infty}\right|}$. Accordingly, we denote a typical element by $t=$ $\left(t_{v}\right)_{v \mid \infty}$. Let $\mathfrak{a}_{\mathbb{C}}$ be its complexification. Let $d t$ be the usual Lebesgue measure on a. Following [BM4], we also equip a with the positive measure $d \mu(t)=\prod_{v \mid \infty} \frac{1}{2} t_{v} \sinh \left(\pi t_{v}\right) d t_{v}$. Let $\mathrm{Pl}: \mathfrak{a} \rightarrow \mathbb{C}$ be defined by

$$
\operatorname{Pl}(t)=\prod_{v \text { real }} 2 \cosh \left(\pi t_{v}\right) \prod_{v \text { complex }} 2 \sinh \left(\pi t_{v}\right) / t_{v} .
$$

4 Note that $d \mu(t) / \mathrm{Pl}(t)$ is the Plancherel measure on the set of spherical tempered representations of $\mathrm{PGL}_{2}\left(F_{\infty}\right)$. Define the logarithm function $\log _{F}: F_{\infty}^{\times} \rightarrow \mathfrak{a}$ by

$$
\log _{F}(x)=\left(\log \left|x_{v}\right|\right)_{v \mid \infty} \text {. }
$$

Bessel kernel and Bessel transform. Let $M>2, N>6$. We set $\mathscr{H}(M, N)$ to be the space of functions $h: \mathfrak{a} \rightarrow \mathbb{C}$ that are of the following form,

$$
h(t)=\prod_{v \mid \infty} h_{v}\left(t_{v}\right)
$$

where each $h_{v}: \mathbb{R} \rightarrow \mathbb{C}$ extends to an even holomorphic function on the strip $\{s=t+i \sigma:$ $|\operatorname{Im} s| \leqslant M\}$ such that, on the horizontal $\operatorname{line} \operatorname{Im} s=\sigma(|\sigma| \leqslant M)$, we have uniformly

$$
h_{v}(t+i \sigma) \ll e^{-\pi|t|}(|t|+1)^{-N} .
$$
$\mathbb{C}^{\left|S_{\infty}\right|}$

We define the Bessel kernel $\mathscr{B}: F_{\infty}^{\times} \times \mathfrak{a}_{\mathbb{C}} \rightarrow \mathbb{C}$ as follows. For $x \in F_{\infty}^{\times}$and $v \in \mathfrak{a}_{\mathbb{C}}=$

$$
\mathscr{B}(x, v)=\prod_{v \mid \infty} B_{v}\left(x_{v}, v_{v}\right)
$$

${ }^{4}$ In $\S 6.7 .2$ and 6.7.3 of [Ven2], the $1 / 2$ in (106) and (112) should be removed, so we have $2 \cosh \left(\pi t_{v}\right)$ here instead of $\cosh \left(\pi t_{v}\right)$. 
where

$$
\begin{aligned}
& B_{v}\left(x_{v}, v_{v}\right)=\frac{1}{\sin \left(\pi v_{v}\right)}\left(J_{-2 v_{v}}\left(4 \pi \sqrt{x_{v}}\right)-J_{2 v_{v}}\left(4 \pi \sqrt{x_{v}}\right)\right), \\
& B_{v}\left(-x_{v}, v_{v}\right)=\frac{1}{\sin \left(\pi v_{v}\right)}\left(I_{-2 v_{v}}\left(4 \pi \sqrt{x_{v}}\right)-I_{2 v_{v}}\left(4 \pi \sqrt{x_{v}}\right)\right)=\frac{4 \cos \left(\pi v_{v}\right)}{\pi} K_{2 v_{v}}\left(4 \pi \sqrt{x_{v}}\right),
\end{aligned}
$$

if $v$ is real and $x_{v}>0$, and

$$
B_{v}\left(x_{v}, v_{v}\right)=\frac{1}{\sin \left(\pi v_{v}\right)}\left(J_{-v_{v}}\left(4 \pi \sqrt{x_{v}}\right) J_{-v_{v}}\left(4 \pi \sqrt{\bar{x}_{v}}\right)-J_{v_{v}}\left(4 \pi \sqrt{x_{v}}\right) J_{v_{v}}\left(4 \pi \sqrt{\bar{x}_{v}}\right)\right),
$$

if $v$ is complex; by definition, we have

$$
B_{v}\left(x_{v}, v_{v}\right)=\boldsymbol{J}_{v_{v}}\left(\sqrt{x_{v}}\right) .
$$

REMARK 1.5. For complex $v$, our definition of $B_{v}\left(x_{v}, v_{v}\right)$ is slightly different from that of [Ven2], in which he uses $I_{v}(x)$ instead of $J_{v}(x)$. But we have the formulae $I_{v}(x)=$ $e^{-\frac{1}{2} \pi i v} J_{v}(i x)$ and $I_{v}(x)=e^{\frac{1}{2} \pi i v} J_{v}(-i x)$ (see [Wat, 3.7 (2)] and it is understood that $i=e^{\frac{1}{2} \pi i}$ and $\left.-i=e^{-\frac{1}{2} \pi i}\right)$. So the difference is only up to the sign of $x_{v}$. This sign difference however would cause a little inconsistency between the Kuznetsov formula for $\mathrm{SL}_{2}(\mathbb{C})$ in $[\mathbf{M W}]$ (or

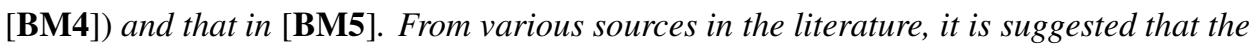
latter (and hence the formula of $B_{v}\left(x_{v}, v_{v}\right)$ here in terms of $J_{v}(x)$ ) should be the correct one.

Let $h(t)$ be a test function on a belonging to $\mathscr{H}(M, N)$ and define its Bessel integral transform $\varphi: F_{\infty}^{\times} \rightarrow \mathbb{C}$ by

$$
\varphi(x)=\int_{\mathfrak{a}} h(t) \mathscr{B}(x, i t) d \mu(t) .
$$

This Bessel transform arises in the Kuznetsov-Bruggeman-Miatello trace formula as its Archimedean component.

Proposition 7 of Venkatesh. The following theorem was proven for totally real $F$ by Venkatesh [Ven2, Proposition 7], as the main ingredient in the Archimedean theory of his work. He mentioned that at the time he had not managed to accomplish the general case. Given this theorem, the main results of Venkatesh for $\mathrm{Sym}^{2}$ may be now extended to arbitrary number field $F$.

Theorem 1.6 (Proposition 7 of Venkatesh). Let $F$ be a number field. Suppose that $h(t) \in \mathscr{H}(M, N)$ and $\varphi(x)$ is the Bessel transform of $h(t)$ defined by (1.31). Let $\mathrm{Pl}: \mathfrak{a} \rightarrow \mathbb{C}$ be defined as in (1.29). Define $\widehat{h \cdot \mathrm{Pl}}: \mathfrak{a} \rightarrow \mathbb{C}$ to be the Fourier transform of $h(t) \mathrm{Pl}(t)$, so for $v \in \mathfrak{a}$,

$$
\widehat{h \cdot \mathrm{Pl}}(v)=\int_{\mathfrak{a}} e^{i\langle t, v\rangle} h(t) \mathrm{Pl}(t) d t,
$$

where $\langle t, v\rangle=\sum_{v \mid \infty} t_{v} v_{v}$. Defining $\log _{F}: F_{\infty}^{\times} \rightarrow \mathfrak{a}$ as in $(1.30$, we have

$$
\widehat{h \cdot \operatorname{Pl}}\left(\log _{F}|\kappa|\right)=\int_{F_{\infty}} \varphi\left(\frac{1}{2+\kappa+\kappa^{-1}} x^{2}\right) \psi_{\infty}(x) \frac{d x}{\operatorname{Norm}(x)} ;
$$

the convergence is guaranteed as long as $M, N$ are sufficiently large.

Since the situation in the presence of multiple Archimedean places is a "product" of situations involving just one Archimedean place, it suffices to check it in the case that 
$F_{\infty}=\mathbb{R}$ or $F_{\infty}=\mathbb{C}$. The case $F_{\infty}=\mathbb{R}$ has already been settled by Venkatesh (see also $\$ 1.1$. The case $F_{\infty}=\mathbb{C}$ will be proven in $\$ 6$ by using our regularized Weber-Schafheitlin integral formula for $\boldsymbol{J}_{\mu}(z)$ in Theorem 1.4

Corollary 1.7. The main results of Venkatesh on Beyond Endoscopy for $\mathrm{Sym}^{2}$ on $\mathrm{GL}_{2}$, in particular his Proposition 2 and Theorem 1, are valid over an arbitrary number field $F$.

ACKNOWLEDGEMENTS. I thank all the participants of my analytic number theory seminar, especially, Dongwen Liu and Zhicheng Wang, at Zhejiang University in the autumn of 2018-Venkatesh's paper was the first one we studied in the seminar. I thank Roman Holowinsky and Akshay Venkatesh for their comments. I also thank the referees for careful readings and helpful comments.

\section{Preliminaries}

\subsection{Classical Bessel functions.}

2.1.1. Basic properties of $J_{v}(z), H_{v}^{(1)}(z)$ and $H_{v}^{(2)}(z)$. Let $v$ be a complex number. Let $J_{v}(z), H_{v}^{(1,2)}(z)$ denote the Bessel function of the first kind and the Hankel functions of order $v$. They all satisfy the Bessel differential equation

$$
z^{2} \frac{d^{2} w}{d z^{2}}(z)+z \frac{d w}{d z}(z)+\left(z^{2}-v^{2}\right) w(z)=0 .
$$

The function $J_{v}(z)$ is defined by the series (see [Wat $\left.3.1(8)\right]$ )

$$
J_{v}(z)=\sum_{n=0}^{\infty} \frac{(-)^{n}(z / 2)^{v+2 n}}{n ! \Gamma(v+n+1)} .
$$

When $v \neq-1,-2,-3, \ldots$, it follows from [Wat 3.13 (1)] that

$$
\left|J_{v}(z)\right|=\frac{(z / 2)^{v}}{\Gamma(v+1)}\left(1+O_{v}\left(|z|^{2}\right)\right), \quad|z| \ll 1,
$$

and, together with the Bessel differential equation and the recurrence formula [Wat, 3.2 (4)],

$$
z J_{v}^{\prime}(z)=v J_{v}(z)-z J_{v+1}(z)
$$

that

$$
z^{r}(d / d z)^{r} J_{v}(z) \ll_{r, v}\left|z^{v}\right|, \quad|z| \ll 1,
$$

with the implied constants uniformly bounded when $v$ lies in a given compact subset of $\mathbb{C} \backslash\{-1,-2,-3, \ldots\}$.

We have the following connection formulae (see [Wat $3.61(1,2)]$ )

$$
J_{v}(z)=\frac{H_{v}^{(1)}(z)+H_{v}^{(2)}(z)}{2}, \quad J_{-v}(z)=\frac{e^{\pi i v} H_{v}^{(1)}(z)+e^{-\pi i v} H_{v}^{(2)}(z)}{2} .
$$

According to [Wat $7.2(1,2)]$ and [Olv 7.13.1], we have Hankel's expansion of $H_{v}^{(1)}(z)$ and $H_{v}^{(2)}(z)$ as follows,

$$
H_{v}^{(1)}(z)=\left(\frac{2}{\pi z}\right)^{\frac{1}{2}} e^{i\left(z-\frac{1}{2} \pi v-\frac{1}{4} \pi\right)}\left(\sum_{n=0}^{N-1} \frac{(-)^{n} \cdot(v, n)}{(2 i z)^{n}}+E_{N}^{(1)}(z)\right),
$$




$$
H_{v}^{(2)}(z)=\left(\frac{2}{\pi z}\right)^{\frac{1}{2}} e^{-i\left(z-\frac{1}{2} \pi v-\frac{1}{4} \pi\right)}\left(\sum_{n=0}^{N-1} \frac{(v, n)}{(2 i z)^{n}}+E_{N}^{(2)}(z)\right),
$$

with $(v, n)=\Gamma(v+n+1 / 2) / n ! \Gamma(v-n+1 / 2)$, of which (2.6) is valid when $z$ is such that $-\pi+\delta \leqslant \arg z \leqslant 2 \pi-\delta$, and (2.7) when $-2 \pi+\delta \leqslant \arg z \leqslant \pi-\delta, \delta$ being any positive acute angle, and

$$
z^{r}(d / d z)^{r} E_{N}^{(1,2)}(z) \ll_{\delta, r, N, v} 1 /|z|^{N}
$$

for $|z| \gg 1$ and $\arg z$ in the range indicated as above. In view of the error bounds in 7.13.1], the dependence of the implied constant on $v$ is uniform in any given compact set.

2.1.2. Some integral formulae. When the order $v=m$ is an integer, we have the integral representation of Bessel for $J_{m}(z)$ as follows (see [Wat 2.2 (1)]),

$$
i^{m} J_{m}(z)=(-i)^{m} J_{-m}(z)=\frac{1}{2 \pi} \int_{0}^{2 \pi} e^{i z \cos \phi-i m \phi} d \phi .
$$

We have the following formula due to Weber, Sonine and Schafheitlin (see [Wat 13.24 (1)] and [GR, 6.561 14]),

$$
\int_{0}^{\infty} \frac{J_{v}(x) d x}{x^{v-\mu+1}}=\frac{\Gamma(\mu / 2)}{2^{v-\mu+1} \Gamma(v-\mu / 2+1)}
$$

in which $0<\operatorname{Re} \mu<\operatorname{Re} v+\frac{3}{2}$ (this is the domain of convergence in [GR 6.561 14], while it is literally $0<\operatorname{Re} \mu<\operatorname{Re} v+\frac{1}{2}$ in [Wat 13.24], for Watson only considers the domain of absolute convergence).

2.2. Preliminaries on the Bessel function $\boldsymbol{J}_{\mu, d}(z)$. Replacing $d / d z$ by $\partial / \partial z$, we denote by $\nabla_{v}$ the differential operator that occurs in (2.1), namely,

$$
\nabla_{v}=z^{2} \frac{\partial^{2}}{\partial z^{2}}+z \frac{\partial}{\partial z}+z^{2}-v^{2}
$$

Its conjugation will be denoted by $\bar{\nabla}_{v}$,

$$
\bar{\nabla}_{v}=\bar{z}^{2} \frac{\partial^{2}}{\partial \bar{z}^{2}}+\bar{z} \frac{\partial}{\partial \bar{z}}+\bar{z}^{2}-v^{2} .
$$

From the definition of $\boldsymbol{J}_{\mu, d}(z)$ as in $1.18,1.19$, we infer that

$$
\nabla_{\mu+d}\left(\boldsymbol{J}_{\mu, d}(z / 4 \pi)\right)=0, \quad \bar{\nabla}_{\mu-d}\left(\boldsymbol{J}_{\mu, d}(z / 4 \pi)\right)=0 .
$$

Recall the definition of $\boldsymbol{R}_{\mu, d}(z)$ given by $(1.24,1.25)$. Suppose at the moment that $|z| \leqslant 2$, say. It follows from (2.3) that if $\mu$ is not an integer, then

$$
\boldsymbol{J}_{\mu, d}(z)-\boldsymbol{R}_{\mu, d}(z) \ll \mu,\left.d|| z\right|^{2-2 \mu}|+||z|^{2+2 \mu} \mid .
$$

Some calculations by the formulae of $\left.\left(\partial J_{v}(z) / \partial v\right)\right|_{v= \pm m}(m=0,1,2, \ldots)$ in [Wat $\$ 3.52$ $(1,2)]$ would imply that in the non-generic case when $\mu$ is an integer,

$$
\boldsymbol{J}_{\mu, d}(z)-\boldsymbol{R}_{\mu, d}(z) \ll_{\mu, d}|z|^{2-2|\mu|} \log (4 /|z|) .
$$

It will be convenient to unify (2.14) and (2.15) in a slightly weaker form as follows,

$$
\boldsymbol{J}_{\mu, d}(z)-\boldsymbol{R}_{\mu, d}(z) \ll \mu, d, \lambda|z|^{2-2 \lambda},
$$

with $\lambda=|\operatorname{Re} \mu|$ if $\mu$ is not an integer and $\lambda>|\operatorname{Re} \mu|$ if otherwise. Further, we have

$$
z^{r} \bar{z}^{s}(\partial / \partial z)^{r}(\partial / \partial \bar{z})^{s} \boldsymbol{J}_{\mu, d}(z) \ll_{r, s, \mu, d, \lambda}|z|^{-2 \lambda} \text {. }
$$


For example, in the generic case, this is a direct consequence of the bounds in (2.4).

In view of the connection formulae in (2.5), we have another expression of $\boldsymbol{J}_{\mu, d}(z)$ in terms of Hankel functions,

$$
\boldsymbol{J}_{\mu, d}(z)=\frac{i}{2}\left(e^{\pi i \mu} H_{\mu, d}^{(1)}(4 \pi z)-e^{-\pi i \mu} H_{\mu, d}^{(2)}(4 \pi z)\right),
$$

with the definition

$$
H_{\mu, d}^{(1,2)}(z)=H_{\mu+d}^{(1,2)}(z) H_{\mu-d}^{(1,2)}(\bar{z}) .
$$

The reader should be warned that the product in (2.19) is not well-defined as function on $\mathbb{C} \backslash\{0\}$. By (2.6)-(2.8), we may write

$$
\boldsymbol{J}_{\mu, d}(z)=e(4 \operatorname{Re} z) \boldsymbol{W}(z)+e(-4 \operatorname{Re} z) \boldsymbol{W}(-z)+\boldsymbol{E}_{N}(z),
$$

where $\boldsymbol{W}(z)$ and $\boldsymbol{E}_{N}(z)$ are real analytic functions on $\mathbb{C} \backslash\{0\}$ satisfying

$$
\begin{aligned}
& z^{r} \bar{z}^{s}(\partial / \partial z)^{r}(\partial / \partial \bar{z})^{s} \boldsymbol{W}(z) \ll_{r, s, N, \mu, d} 1 /|z|, \\
& (\partial / \partial z)^{r}(\partial / \partial \bar{z})^{s} \boldsymbol{E}_{N}(z) \ll_{r, s, N, \mu, d} 1 /|z|^{N+1},
\end{aligned}
$$

for $|z| \geqslant 1$.

2.3. The hypergeometric function. Our reference for the hypergeometric function is Chapter II of [MOS].

The hypergeometric function ${ }_{2} F_{1}(a, b ; c ; z)$ is defined by the Gauss series

$$
{ }_{2} F_{1}(a, b ; c ; z)=\frac{\Gamma(c)}{\Gamma(a) \Gamma(b)} \sum_{n=0}^{\infty} \frac{\Gamma(a+n) \Gamma(b+n)}{\Gamma(c+n) n !} z^{n}
$$

within its circle of convergence $|z|<1$, and by analytic continuation elsewhere. The series is absolutely convergent on the unit circle $|z|=1$ if $\operatorname{Re}(a+b-c)<0$. The function ${ }_{2} F_{1}(a, b ; c ; z)$ is a single-valued analytic function of $z$ in the complex plane with a branch cut along the positive real axis from 1 to $\infty$. Moreover, ${ }_{2} F_{1}(a, b ; c ; z)$ is analytic in $a, b$ and $c$, except for $c=0,-1,-2, \ldots$.

The hypergeometric differential equation satisfied by ${ }_{2} F_{1}(a, b ; c ; z)$ is as follows,

$$
z(1-z) \frac{d^{2} w}{d z^{2}}(z)+(c-(a+b+1) z) \frac{d w}{d z}(z)-a b w(z)=0 .
$$

It has three regular singular points $z=0,1, \infty$. In the generic case when none of $c, a-b$ and $c-a-b$ is an integer, two linearly independent solutions of (2.24) in the vicinity of $z=\infty$ are given by

$$
\begin{aligned}
& G_{1}(a, b ; c ; z)=z^{-a}{ }_{2} F_{1}(a, a-c+1 ; a-b+1 ; 1 / z), \\
& G_{2}(a, b ; c ; z)=z^{-b}{ }_{2} F_{1}(b, b-c+1 ; b-a+1 ; 1 / z) .
\end{aligned}
$$

Finally, we record here the transformation formula with respect to $z \rightarrow 1 / z$ (see [MOS $\S 2.4 .1])$,

$$
\begin{array}{r}
{ }_{2} F_{1}(a, b ; c ; z)= \\
+\frac{\Gamma(c) \Gamma(b-a)}{\Gamma(b) \Gamma(c-a)}(-z)^{-a}{ }_{2} F_{1}(a, a-c+1 ; a-b+1 ; 1 / z) \\
+\frac{\Gamma(c) \Gamma(a-b)}{\Gamma(a) \Gamma(c-b)}(-z)^{-b}{ }_{2} F_{1}(b, b-c+1 ; b-a+1 ; 1 / z), \\
|\arg (-z)|<\pi, a-b \neq \pm m, m=0,1,2, \ldots
\end{array}
$$


2.4. A lemma on oscillatory integrals. The following lemma will be very useful in our later analysis.

Lemma 2.1. Let $a, b$ and $\theta$ be real numbers such that $|b|<|a|$. Let $0<A<B \leqslant \infty$, $\gamma<\frac{1}{2}$, and $M$ be a positive integer. Suppose that $f(z)$ is a smooth function on $\mathbb{C}$ which is supported on the annulus $|z| \in[A, B]$ and satisfies

$$
x^{r}(\partial / \partial x)^{r}(\partial / \partial \phi)^{s} f\left(x e^{i \phi}\right) \ll_{r, s} x^{2 \gamma-2}
$$

for all nonnegative integers $r, s$ such that $r+s \leqslant 2 M$ (equivalently,

$$
z^{r} \bar{z}^{s}(\partial / \partial z)^{r}(\partial / \partial \bar{z})^{s} f(z) \ll_{r, s}|z|^{2 \gamma-2}
$$

for all $r+s \leqslant 2 M)$. Define

$$
I(a, b, \theta)=\int_{0}^{2 \pi} \int_{0}^{\infty} f\left(x e^{i \phi}\right) e(x(a \cos (\phi+\theta)+b \cos \phi)) x d x d \phi
$$

Then the integral I $(a, b, \theta)$ is convergent after integration by parts (it is already absolutely convergent if $\gamma<0$ ) and

$$
I(a, b, \theta) \ll_{M, \gamma} \frac{1}{A^{2 M-2 \gamma}(|a|-|b|)^{2 M}},
$$

with the convergence and the implied constant uniform for $\gamma$ in a compact set. Moreover, the integral $I(a, b, \theta)$ gives rise to a continuous function in $(a, \theta)$ (the continuity extends to all real values of a if $\gamma<0$ ).

Proof. Put $p(x, \phi ; a, b, \theta)=x(a \cos (\phi+\theta)+b \cos \phi)$. Define the differential operator $\mathrm{D}=(\partial / \partial x)^{2}+(\partial / \partial x) / x+(\partial / \partial \phi)^{2} / x^{2}$ so that

$$
\mathrm{D}(e(p(x, \phi ; a, b, \theta)))=-4 \pi^{2}\left(a^{2}+b^{2}+2 a b \cos \theta\right) \cdot e(p(x, \phi ; a, b, \theta)) .
$$

Note that $\mathrm{D}$ is self-adjoint. In view of the conditions on $f(z)$, it follows from an application of the partial integration with respect to $\mathrm{D}$ that

$$
I(a, b, \theta)=-\frac{1}{4 \pi^{2}\left(a^{2}+b^{2}+2 a b \cos \theta\right)} \int_{0}^{2 \pi} \int_{0}^{\infty} \mathrm{D} f\left(x e^{i \phi}\right) e(p(x, \phi ; a, b, \theta)) x d x d \phi .
$$

Since $x \mathrm{D} f\left(x e^{i \phi}\right) \ll x^{2 \gamma-3}$, the integral above is absolutely convergent as $\gamma<\frac{1}{2}$. Repeating the partial integration above $M$ times and then bounding the resulting integral trivially, along with $a^{2}+b^{2}+2 a b \cos \theta \geqslant(|a|-|b|)^{2}$ and $x \mathrm{D}^{M} f\left(x e^{i \phi}\right) \ll_{M} x^{2 \gamma-2 M-1}$, we obtain the estimates for $I(a, b, \theta)$ in the lemma. Finally, the continuity of $I(a, b, \theta)$ is obvious.

Q.E.D.

\section{More on the hypergeometric function}

We shall be concerned with the hypergeometric differential equation for

$$
a=\frac{\rho+v}{2}, \quad b=\frac{\rho-v}{2}, \quad c=\frac{1}{2} .
$$

Define the corresponding hypergeometric differential operator

$$
\nabla_{\rho, v}=4 z(1-z) \frac{\partial^{2}}{\partial z^{2}}+2(1-2(\rho+1) z) \frac{\partial}{\partial z}-\left(\rho^{2}-v^{2}\right),
$$


and its conjugate

$$
\bar{\nabla}_{\rho, v}=4 \bar{z}(1-\bar{z}) \frac{\partial^{2}}{\partial \bar{z}^{2}}+2(1-2(\rho+1) \bar{z}) \frac{\partial}{\partial \bar{z}}-\left(\rho^{2}-v^{2}\right) .
$$

For the choice of $a, b, c$ in (3.1) such that neither $v$ nor $\rho-\frac{1}{2}$ is an integer, we denote

$$
G_{\rho, v}^{(1)}(z)=G_{1}(a, b ; c ; z), \quad G_{\rho, v}^{(2)}(z)=G_{2}(a, b ; c ; z) .
$$

Let $F_{\rho, v}^{(1)}(z)$ and $F_{\rho, v}^{(2)}(z)$ be defined as in (1.21). Note that their hypergeometric series expansions are absolutely convergent for all $|z| \leqslant 1$ if $\operatorname{Re} \rho<\frac{1}{2}$. Further, we have

$$
G_{\rho, v}^{(1)}(z)=z^{-\frac{1}{2}(\rho+v)} F_{\rho, v}^{(1)}(1 / z), \quad G_{\rho, v}^{(2)}(z)=z^{-\frac{1}{2}(\rho-v)} F_{\rho, v}^{(2)}(1 / z) .
$$

Lemma 3.1. Let $d$ be an integer. Let $\rho, \mu$ be such that $\rho-\frac{1}{2}$ is not an integer and that $|\operatorname{Re} \mu|<\frac{1}{2}$ and $\mu \neq 0$. Let $f(z)$ be a continuous function on the complex plane which is a solution of the following two differential equations,

$$
\nabla_{\rho, \mu+d} w=0, \quad \bar{\nabla}_{\rho, \mu-d} w=0,
$$

with the differential operators $\nabla_{\rho, \mu+d}$ and $\bar{\nabla}_{\rho, \mu-d}$ defined as in (3.2) and (3.3). Suppose further that $f(z)$ admits the following asymptotic,

$$
f(z) \sim c_{1} z^{-\frac{1}{2}(\rho+\mu+d)} \bar{z}^{-\frac{1}{2}(\rho+\mu-d)}+c_{2} z^{-\frac{1}{2}(\rho-\mu-d)} \bar{z}^{-\frac{1}{2}(\rho-\mu+d)}, \quad|z| \rightarrow \infty .
$$

Then

$$
f(z)=c_{1} G_{\rho, \mu+d}^{(1)}(z) G_{\rho, \mu-d}^{(1)}(\bar{z})+c_{2} G_{\rho, \mu+d}^{(2)}(z) G_{\rho, \mu-d}^{(2)}(\bar{z})
$$

for all $z$ in the complex plane.

Proof. By the theory of differential equations, it follows from (3.6) that $f(z)$ may be uniquely written as a linear combination of $G_{\rho, \mu+d}^{(k)}(z) G_{\rho, \mu-d}^{(l)}(\bar{z})$, with $k, l=1,2$, namely,

$$
f(z)=\sum_{k, l=1,2} c_{k l} G_{\rho, \mu+d}^{(k)}(z) G_{\rho, \mu-d}^{(l)}(\bar{z}),
$$

for all $z$ away from the branch cut from 0 to 1 .

In view of (2.25) and (3.4), it is clear that $G_{\rho, \mu+d}^{(1)}(z) G_{\rho, \mu-d}^{(1)}(\bar{z})$ and $G_{\rho, \mu+d}^{(2)}(z) G_{\rho, \mu-d}^{(2)}(\bar{z})$ are both single-valued real analytic functions when $|z|>1$, and so are the quotient of $G_{\rho, \mu+d}^{(1)}(z) G_{\rho, \mu-d}^{(2)}(\bar{z})$ and $(z /|z|)^{-\mu}$ and that of $G_{\rho, \mu+d}^{(2)}(z) G_{\rho, \mu-d}^{(1)}(\bar{z})$ and $(z /|z|)^{\mu}$. Since $\mu$ is not an integer, the functions $(z /|z|)^{-\mu}$ and $(z /|z|)^{\mu}$ are not single-valued. Choosing $\arg z=0,2 \pi$, it follows from simple considerations that any nontrivial linear combination of $G_{\rho, \mu+d}^{(1)}(z) G_{\rho, \mu-d}^{(2)}(\bar{z})$ and $G_{\rho, \mu+d}^{(2)}(z) G_{\rho, \mu-d}^{(1)}(\bar{z})$ is not independent on $\arg z$ modulo $2 \pi$. So we must have $c_{12}=c_{21}=0$.

Next, we need to prove that $c_{11}=c_{1}$ and $c_{22}=c_{2}$. For this, we deduce from 2.25) and (3.4) that

$$
\begin{aligned}
& G_{\rho, \mu+d}^{(1)}(z) G_{\rho, \mu-d}^{(1)}(\bar{z})=|z|^{-\rho-\mu}(\bar{z} /|z|)^{d}+O\left(\left.|| z\right|^{-\rho-\mu-1} \mid\right), \\
& G_{\rho, \mu+d}^{(2)}(z) G_{\rho, \mu-d}^{(2)}(\bar{z})=|z|^{-\rho+\mu}(z /|z|)^{d}+O\left(\left.|| z\right|^{-\rho+\mu-1} \mid\right),
\end{aligned}
$$

as $|z| \rightarrow \infty$. Since $|\operatorname{Re} \mu|<\frac{1}{2}$, the two error terms have order strictly lower than the least order of the two main terms. This forces $c_{11}=c_{1}$ and $c_{22}=c_{2}$ in order for $f(z)$ to have the prescribed asymptotic in (3.7). 
It is now proven that the identity (3.8) is valid except on the branch cut from 0 to 1 . However $f(z)$ is continuous on the whole complex plane, the identity may be extended to the branch cut.

Q.E.D.

We remark that the continuity of $f(z)$ is a very strong condition (it is not even clear now whether exists such a continuous solution $f(z)$ of the differential equations). It does not only force $c_{12}=c_{21}=0$ in the proof above but also implies that the ratio $c_{1} / c_{2}$ has to be unique as neither $G_{\rho, \mu+d}^{(1)}(z) G_{\rho, \mu-d}^{(1)}(\bar{z})$ nor $G_{\rho, \mu+d}^{(2)}(z) G_{\rho, \mu-d}^{(2)}(\bar{z})$ is single-valued in $|z|<1$. Indeed, the following proposition demonstrates that the ratio must be equal to $4^{-\mu} C_{\mu, d}^{\rho} / 4^{\mu} C_{-\mu,-d}^{\rho}$. This proposition however is not required in our proof of Theorem 1.1

Proposition 3.2. Let $C_{\mu, d}^{\rho}$ be defined by (1.20). Let $G_{\rho, v}^{(1)}(z)$ and $G_{\rho, v}^{(2)}(z)$ be given by (1.21) and (3.5). Define

$$
E_{\rho, v}^{(1)}(z)={ }_{2} F_{1}\left(\frac{\rho+v}{2}, \frac{\rho-v}{2} ; \frac{1}{2} ; z\right), E_{\rho, v}^{(2)}(z)={ }_{2} F_{1}\left(\frac{\rho+v+1}{2}, \frac{\rho-v+1}{2} ; \frac{3}{2} ; z\right) .
$$

5 We have

$$
\begin{aligned}
& 4^{-\mu} C_{\mu, d}^{\rho} G_{\rho, \mu+d}^{(1)}(z) G_{\rho, \mu-d}^{(1)}(\bar{z})+4^{\mu} C_{-\mu,-d}^{\rho} G_{\rho, \mu+d}^{(2)}(z) G_{\rho, \mu-d}^{(2)}(\bar{z}) \\
= & B_{\rho, \mu, d} A_{\rho, \mu, d}^{(1)} E_{\rho, \mu+d}^{(1)}(z) E_{\rho, \mu-d}^{(1)}(\bar{z})+B_{\rho, \mu, d+1} A_{\rho, \mu, d}^{(2)} \cdot 4|z| E_{\rho, \mu+d}^{(2)}(z) E_{\rho, \mu-d}^{(2)}(\bar{z}),
\end{aligned}
$$

for $\arg z \in(0,2 \pi)$, with

$$
\begin{gathered}
B_{\rho, \mu, d}=\frac{(-)^{d} \cos (\pi \mu)-\cos (\pi \rho)}{4 \pi^{2 \rho+2}}, \\
A_{\rho, \mu, d}^{(1)}=\Gamma\left(\frac{\rho+\mu+d}{2}\right) \Gamma\left(\frac{\rho+\mu-d}{2}\right) \Gamma\left(\frac{\rho-\mu-d}{2}\right) \Gamma\left(\frac{\rho-\mu+d}{2}\right),
\end{gathered}
$$

and similarly

$$
A_{\rho, \mu, d}^{(2)}=\Gamma\left(\frac{1+\rho+\mu+d}{2}\right) \Gamma\left(\frac{1+\rho+\mu-d}{2}\right) \Gamma\left(\frac{1+\rho-\mu-d}{2}\right) \Gamma\left(\frac{1+\rho-\mu+d}{2}\right) .
$$

Moreover, for $\operatorname{Re} \rho<\frac{1}{2}$, the combinations on both sides of (3.10) give rise to a continuous function of $z$ on the whole complex plane, real-analytic except at $z=1$.

Proof. By the transformation law (2.26), we have

$$
\begin{aligned}
& G_{\rho, v}^{(1)}(z)=\frac{\sqrt{\pi} \Gamma(1+v) e^{-\frac{1}{2} \pi i(\rho+v)} E_{\rho, v}^{(1)}(z)}{\Gamma((1+\rho+v) / 2) \Gamma((2-\rho+v) / 2)}+\frac{2 \sqrt{\pi} \Gamma(1+v) e^{-\frac{1}{2} \pi i(\rho+v+1)} \sqrt{z} E_{\rho, v}^{(2)}(z)}{\Gamma((\rho+v) / 2) \Gamma((1-\rho+v) / 2)}, \\
& G_{\rho, v}^{(2)}(z)=\frac{\sqrt{\pi} \Gamma(1-v) e^{-\frac{1}{2} \pi i(\rho-v)} E_{\rho, v}^{(1)}(z)}{\Gamma((1+\rho-v) / 2) \Gamma((2-\rho-v) / 2)}+\frac{2 \sqrt{\pi} \Gamma(1-v) e^{-\frac{1}{2} \pi i(\rho-v+1)} \sqrt{z} E_{\rho, v}^{(2)}(z)}{\Gamma((\rho-v) / 2) \Gamma((1-\rho-v) / 2)},
\end{aligned}
$$

for $\arg z \in(0,2 \pi)$. For $G_{\rho, v}^{(1)}(\bar{z})$ and $G_{\rho, v}^{(1)}(\bar{z})$, the formulae are similar but in a subtle waythe factors like $e^{-\frac{1}{2} \pi i(\rho+v)}$ should be replaced by $e^{\frac{1}{2} \pi i(\rho+v)}$. This is because, in view of the conditions in (2.26), we need to let $|\arg (-1 / \bar{z})|<\pi$ and hence $-1=e^{-i \pi}$.

Applying these to the left hand side of (3.10). By the duplication formula for the gamma function, we find that, up to sign, the coefficients of $E_{\rho, \mu+d}^{(1)}(z) \cdot \sqrt{\bar{z}} E_{\rho, \mu-d}^{(2)}(\bar{z})$ and

${ }^{5}$ Note that, according to [MOS $\left.\$ 2.2\right], E_{\rho, v}^{(1)}(z)$ and $\sqrt{z} E_{\rho, v}^{(2)}(z)$ form a system of linearly independent solutions of the hypergeometric equation with $a, b, c$ as in 3.1 in the vicinity of the singular point $z=0$. 


$$
\begin{aligned}
& \sqrt{z} E_{\rho, \mu+d}^{(2)}(z) \cdot E_{\rho, \mu-d}^{(1)}(\bar{z}) \text { are both equal to } \\
& \begin{aligned}
\frac{i^{2 d-1}}{2 \pi^{2 \rho} \sin (\pi \mu)}( & \frac{\sin (\pi(\rho+\mu)) \Gamma((\rho+\mu+d) / 2) \Gamma((1+\rho+\mu-d) / 2)}{\Gamma((2-\rho+\mu+d) / 2) \Gamma((1-\rho+\mu-d) / 2)} \\
& \left.-\frac{\sin (\pi(\rho-\mu)) \Gamma((\rho-\mu-d) / 2) \Gamma((1+\rho-\mu+d) / 2)}{\Gamma((2-\rho-\mu-d) / 2) \Gamma((1-\rho-\mu+d) / 2)}\right),
\end{aligned}
\end{aligned}
$$

which in turn is equal to zero by Euler's reflection formula for the gamma function. We obtain (3.10) by calculating the coefficients of $E_{\rho, v}^{(1)}(z) E_{\rho, v}^{(1)}(\bar{z})$ and $|z| E_{\rho, v}^{(2)}(z) E_{\rho, v}^{(2)}(\bar{z})$ in a similar way.

It was noted before that the left hand side of (3.10) gives rise to a single-valued continuous function on $|z| \geqslant 1$, real-analytic in the interior $|z|>1$, provided that $\operatorname{Re} \rho<\frac{1}{2}$. It is also clear that the same is true for the right hand side of $(3.10)$ on $|z| \leqslant 1$. Thus (3.10) is valid and defines a continuous function (real-analytic except at $z=1$ ) on the whole complex plane if $\operatorname{Re} \rho<\frac{1}{2}$.

Q.E.D.

\section{Proof of Theorem 1.1}

Recall that $\boldsymbol{J}_{\mu, d}(z)$ and $\boldsymbol{R}_{\mu, d}(z)$ were defined as in (1.18, 1.19) and (1.24[1.25) respectively. Let

$$
\boldsymbol{F}_{\mu, d}^{\rho}\left(y e^{i \theta}\right)=\int_{0}^{2 \pi} \int_{0}^{\infty} \boldsymbol{J}_{\mu, d}\left(x e^{i \phi}\right) e(-2 x y \cos (\phi+\theta)) x^{2 \rho-1} d x d \phi
$$

and

$$
\boldsymbol{P}_{\mu, d}^{\rho}\left(y e^{i \theta}\right)=\int_{0}^{2 \pi} \int_{0}^{\infty} \boldsymbol{R}_{\mu, d}\left(x e^{i \phi}\right) e(-2 x y \cos (\phi+\theta)) x^{2 \rho-1} d x d \phi .
$$

Since $\boldsymbol{J}_{\mu, d}(z)$ is an even function, the integral $\boldsymbol{F}_{\mu, d}^{\rho}\left(y e^{i \theta}\right)$ is also even in the sense that it is independent on $\theta$ modulo $\pi$.

First of all, the convergence of $\boldsymbol{F}_{\mu, d}^{\rho}\left(y e^{i \theta}\right)$ and $\boldsymbol{P}_{\mu, d}^{\rho}\left(y e^{i \theta}\right)$ may be easily examined with the help of Lemma 2.1, along with (2.17) and (2.20)-(2.22) for the former.

Lemma 4.1. Let the integrals $\boldsymbol{F}_{\mu, d}^{\rho}\left(y e^{i \theta}\right)$ and $\boldsymbol{P}_{\mu, d}^{\rho}\left(y e^{i \theta}\right)$ be defined as above.

(1). $\boldsymbol{F}_{\mu, d}^{\rho}\left(y e^{i \theta}\right)$ is absolutely convergent if $|\operatorname{Re} \mu|<\operatorname{Re} \rho<\frac{1}{2}$, and, for $y>2$, convergent if $|\operatorname{Re} \mu|<\operatorname{Re} \rho<1$, uniformly for $\operatorname{Re} \mu$ and $\operatorname{Re} \rho$ in compact sets.

(2). $\boldsymbol{P}_{\mu, d}^{\rho}\left(y e^{i \theta}\right)$ is convergent for $y>0$ if $|\operatorname{Re} \mu|<\operatorname{Re} \rho<\frac{1}{2}-|\operatorname{Re} \mu|$, uniformly for $\operatorname{Re} \mu$ and $\operatorname{Re} \rho$ in compact sets.

Moreover, both $\boldsymbol{F}_{\mu, d}^{\rho}\left(y e^{i \theta}\right)$ and $\boldsymbol{P}_{\mu, d}^{\rho}\left(y e^{i \theta}\right)$ are continuous functions when $y$ is in the indicated ranges above.

Proof. We partition the integrals $\boldsymbol{F}_{\mu, d}^{\rho}\left(y e^{i \theta}\right)$ and $\boldsymbol{P}_{\mu, d}^{\rho}\left(y e^{i \theta}\right)$ according to a partition of unity $(1-w(x))+w(x) \equiv 1$ for $(0, \infty)=(0,2] \cup[1, \infty)$, say.

By (2.17), it is obvious that the integral

$$
\int_{0}^{2 \pi} \int_{0}^{2}(1-w(x)) \boldsymbol{J}_{\mu, d}\left(x e^{i \phi}\right) e(-2 x y \cos (\phi+\theta)) x^{2 \rho-1} d x d \phi
$$

is convergent if $|\operatorname{Re} \mu|<\operatorname{Re} \rho$; the same statement is true if $\boldsymbol{J}_{\mu, d}$ is substituted by $\boldsymbol{R}_{\mu, d}$. 
Next, we consider the following integral

$$
\int_{0}^{2 \pi} \int_{1}^{\infty} w(x) \boldsymbol{J}_{\mu, d}\left(x e^{i \phi}\right) e(-2 x y \cos (\phi+\theta)) x^{2 \rho-1} d x d \phi
$$

Since $\boldsymbol{J}_{\mu, d}(z)=O(1 /|z|)$ for $|z| \geqslant 1$, this integral is absolutely convergent provided that $\operatorname{Re} \rho<\frac{1}{2}$. According to $(2.20)$, we divide it into three similar integrals which contain $\boldsymbol{W}\left(x e^{i \phi}\right), \boldsymbol{W}\left(-x e^{i \phi}\right)$ and $\boldsymbol{E}_{1}\left(x e^{i \phi}\right)$ respectively. For the first two integrals, we apply Lemma 2.1 with $a=-2 y, b= \pm 4, f(z)=w(|z|) \boldsymbol{W}( \pm z)|z|^{2 \rho-2}$ and $\gamma=\operatorname{Re} \rho-\frac{1}{2}$ (by (2.21)), and it follows that these two integrals are convergent if $\operatorname{Re} \rho<1\left(\gamma<\frac{1}{2}\right)$. By (2.22), the third integral is absolutely convergent when $\operatorname{Re} \rho<1$.

Similarly, applying Lemma2.1 with $a=-2 y, b=0, f(z)=w(|z|) \boldsymbol{R}_{\mu, d}(z)|z|^{2 \rho-2}$ and $\gamma=\operatorname{Re} \rho+|\operatorname{Re} \mu|$, we infer that the integral

$$
\int_{0}^{2 \pi} \int_{1}^{\infty} w(x) \boldsymbol{R}_{\mu, d}\left(x e^{i \phi}\right) e(-2 x y \cos (\phi+\theta)) x^{2 \rho-1} d x d \phi
$$

is convergent provided that $\operatorname{Re} \rho<\frac{1}{2}-|\operatorname{Re} \mu|$.

Q.E.D.

4.1. Asymptotic of $\boldsymbol{F}_{\mu, d}^{\rho}\left(y e^{i \theta}\right)$. In this section, we assume that $\lambda=|\operatorname{Re} \mu|<\frac{1}{4}$, $\beta=\operatorname{Re} \rho$ and $\lambda<\beta<\frac{1}{2}-\lambda$ so that $\boldsymbol{F}_{\mu, d}^{\rho}\left(y e^{i \theta}\right)$ and $\boldsymbol{P}_{\mu, d}^{\rho}\left(y e^{i \theta}\right)$ are both convergent. Moreover, let $\mu \neq 0$ for simplicity.

Our first aim is to prove the following asymptotic of $\boldsymbol{F}_{\mu, d}^{\rho}\left(y e^{i \theta}\right)$,

$$
\boldsymbol{F}_{\mu, d}^{\rho}\left(y e^{i \theta}\right) \sim C_{\mu, d}^{\rho} y^{-2 \mu-2 \rho} e^{-2 i d \theta}+C_{-\mu,-d}^{\rho} y^{2 \mu-2 \rho} e^{2 i d \theta}, \quad y \rightarrow \infty,
$$

in which $C_{\mu, d}^{\rho}$ is defined by (1.20) in Theorem 1.1 It is clear that (4.3) follows from the following two lemmas.

Lemma 4.2. We have

$$
\boldsymbol{F}_{\mu, d}^{\rho}\left(y e^{i \theta}\right)=\boldsymbol{P}_{\mu, d}^{\rho}\left(y e^{i \theta}\right)+o\left(y^{-2 \lambda-2 \beta}\right), \quad y \rightarrow \infty .
$$

Lemma 4.3. We have

$$
\boldsymbol{P}_{\mu, d}^{\rho}\left(y e^{i \theta}\right)=C_{\mu, d}^{\rho} y^{-2 \mu-2 \rho} e^{-2 i d \theta}+C_{-\mu,-d}^{\rho} y^{2 \mu-2 \rho} e^{2 i d \theta} .
$$

Proof of Lemma 4.2 Let $y>2$ be sufficiently large. All the implied constants in our computation will only depend on $\beta, \lambda$ and $d$.

We split $\boldsymbol{F}_{\mu, d}^{\rho}\left(y e^{i \theta}\right)-\boldsymbol{P}_{\mu, d}^{\rho}\left(y e^{i \theta}\right)$ as the sum

$$
\begin{aligned}
\boldsymbol{F}_{\mu, d}^{\rho}\left(y e^{i \theta}\right)- & \boldsymbol{P}_{\mu, d}^{\rho}\left(y e^{i \theta}\right)=\boldsymbol{D}_{\mu, d}^{\rho}\left(y e^{i \theta}\right)+\grave{\boldsymbol{E}}_{\mu, d}^{\rho}\left(y e^{i \theta}\right)+\grave{\boldsymbol{E}}_{\mu, d}^{\rho}\left(y e^{i \theta}\right)-\hat{\boldsymbol{E}}_{\mu, d}^{\rho}\left(y e^{i \theta}\right) \\
= & \int_{0}^{2 \pi} \int_{0}^{2 y^{-\frac{1}{2}}} u(x)\left(\boldsymbol{J}_{\mu, d}\left(x e^{i \phi}\right)-\boldsymbol{R}_{\mu, d}\left(x e^{i \phi}\right)\right) e(-2 x y \cos (\phi+\theta)) x^{2 \rho-1} d x d \phi \\
& +\int_{0}^{2 \pi} \int_{y^{-\frac{1}{2}}}^{2} v(x) \boldsymbol{J}_{\mu, d}\left(x e^{i \phi}\right) e(-2 x y \cos (\phi+\theta)) x^{2 \rho-1} d x d \phi \\
& +\int_{0}^{2 \pi} \int_{1}^{\infty} w(x) \boldsymbol{J}_{\mu, d}\left(x e^{i \phi}\right) e(-2 x y \cos (\phi+\theta)) x^{2 \rho-1} d x d \phi \\
& -\int_{0}^{2 \pi} \int_{y^{-\frac{1}{2}}}^{\infty}(v(x)+w(x)) \boldsymbol{R}_{\mu, d}\left(x e^{i \phi}\right) e(-2 x y \cos (\phi+\theta)) x^{2 \rho-1} d x d \phi
\end{aligned}
$$


where $u(x)+v(x)+w(x) \equiv 1$ is a partition of unity on $(0, \infty)$ such that $u(x), v(x)$ and $w(x)$ are smooth functions supported on $\left(0,2 y^{-\frac{1}{2}}\right],\left[y^{-\frac{1}{2}}, 2\right]$ and $[1, \infty)$, respectively, and that $x^{r} u^{(r)}(x), x^{r} v^{(r)}(x), x^{r} w^{(r)}(x) \ll_{r} 1$.

First, it follows from (2.16) that

$$
\boldsymbol{D}_{\mu, d}^{\rho}\left(y e^{i \theta}\right) \ll \int_{0}^{2 \pi} \int_{0}^{2 y^{-\frac{1}{2}}} x^{2 \beta-2 \lambda+1} d x d \phi \ll y^{\lambda-\beta-1}=y^{-2 \lambda-2 \beta} y^{3 \lambda+\beta-1} .
$$

Since $\beta<\frac{1}{2}-\lambda$ and $\lambda<\frac{1}{4}$, we have $3 \lambda+\beta-1<2 \lambda-\frac{1}{2}<0$ and hence $D_{\mu, d}^{\rho}\left(y e^{i \theta}\right)=$ $o\left(y^{-2 \lambda-2 \beta}\right)$ as desired.

Second, applying Lemma 2.1 with $a=-2 y, b=0, A=y^{-\frac{1}{2}}, B=2, M=1$, $f(z)=v(|z|) \boldsymbol{J}_{\mu, d}(z)|z|^{2 \rho-2}$ and $\gamma=\beta-\lambda$ (see (2.17)), we deduce that

$$
\dot{\boldsymbol{E}}_{\mu, d}^{\rho}\left(y e^{i \theta}\right) \ll y^{(1-\beta+\lambda)-2}=y^{\lambda-\beta-1} .
$$

As above, we also have $\hat{\boldsymbol{E}}_{\mu, d}^{\rho}\left(y e^{i \theta}\right)=o\left(y^{-2 \lambda-2 \beta}\right)$. Likewise, $\hat{\boldsymbol{E}}_{\mu, d}^{\rho}\left(y e^{i \theta}\right)=o\left(y^{-2 \lambda-2 \beta}\right)$.

Third, according to (2.20), we divide $\grave{\boldsymbol{E}}_{\mu, d}^{\rho}\left(y e^{i \theta}\right)$ into three similar integrals which contain $\boldsymbol{W}\left(x e^{i \phi}\right), \boldsymbol{W}\left(-x e^{i \phi}\right)$ and $\boldsymbol{E}_{1}\left(x e^{i \phi}\right)$ respectively. For the first two integrals, we apply Lemma2.1 with $a=-2 y, b= \pm 4, A=1, B=\infty, M=1, f(z)=w(|z|) \boldsymbol{W}( \pm z)|z|^{2 \rho-2}$ and $\gamma=\beta-\frac{1}{2}$ (by (2.21) ), and it follows that these two integrals are both $O\left(y^{-2}\right)$. For the third integral containing $\boldsymbol{E}_{1}\left(x e^{i \phi}\right)$, we apply Lemma 2.1 with $a=-2 y, b=0, A=1$, $B=\infty, M=1, f(z)=w(|z|) \boldsymbol{E}_{1}(z)|z|^{2 \rho-2}$ and $\gamma=\beta$ (by (2.22) $)$, and it follows that the third integral is also $O\left(y^{-2}\right)$. We conclude that

$$
\grave{\boldsymbol{E}}_{\mu, d}^{\rho}\left(y e^{i \theta}\right)=O\left(y^{-2}\right)=o\left(y^{-2 \lambda-2 \beta}\right)
$$

as $\beta<\frac{1}{2}-\lambda$.

Finally, combining the foregoing results, we obtain the asymptotic formula (4.4) in Lemma 4.26

Q.E.D.

The formula (4.5) in Lemma 4.3 is an immediate consequence of the following lemma, applied with $m= \pm 2 d$ and $v=\rho \pm \mu$.

Lemma 4.4. Let $m$ be an integer. Let $-\frac{1}{2}|m|<\operatorname{Re} v<\frac{3}{4}$. We have

$$
\int_{0}^{\infty} \int_{0}^{2 \pi} e(-2 x y \cos (\phi+\theta)) x^{2 v-1} e^{i m \phi} d \phi d x=\left\{\begin{array}{l}
\frac{\sin (\pi v) \Gamma(v+m / 2) \Gamma(v-m / 2)}{(2 \pi)^{2 v} y^{2 v} e^{i m \theta}}, \\
\frac{i \cos (\pi v) \Gamma(v+m / 2) \Gamma(v-m / 2)}{(2 \pi)^{2 v} y^{2 v} e^{i m \theta}},
\end{array}\right.
$$

according as $m$ is even or odd; the integral on the left is convergent as iterated integral for $-\frac{1}{2}|m|<\operatorname{Re} v<\frac{3}{4}$ and as double integral only for $0<\operatorname{Re} v<\frac{1}{2}$.

Proof. The inner integral over $\phi$ may be evaluated by (2.9) so that

$$
\int_{0}^{\infty} \int_{0}^{2 \pi} e(-2 x y \cos (\phi+\theta)) e^{i m \phi} d \phi x^{2 \nu-1} d x=2 \pi(-i)^{|m|} e^{-i m \theta} \int_{0}^{\infty} J_{|m|}(4 \pi x y) x^{2 v-1} d x .
$$

${ }^{6}$ Even more, it may be proven that that $\hat{\boldsymbol{E}}_{\mu, d}^{\rho}\left(y e^{i \theta}\right), \grave{\boldsymbol{E}}_{\mu, d}^{\rho}\left(y e^{i \theta}\right)$ and $\hat{\boldsymbol{E}}_{\mu, d}^{\rho}\left(y e^{i \theta}\right)$ are all arbitrarily small, namely, $O_{A}\left(y^{-A}\right)$ for arbitrary $A>0$. 
In view of (2.10), the integral in the right hand side is equal to

$$
\frac{\Gamma(v+|m| / 2)}{2(2 \pi y)^{2 v} \Gamma(1-v+|m| / 2)}= \begin{cases}\frac{\sin (\pi v) \Gamma(v+|m| / 2) \Gamma(v-|m| / 2)}{2 \pi(2 \pi y)^{2 v} i|m|}, & \text { if } m \text { is even } \\ \frac{\cos (\pi v) \Gamma(v+|m| / 2) \Gamma(v-|m| / 2)}{2 \pi(2 \pi y)^{2 v} i^{|m|+1}}, & \text { if } m \text { is odd }\end{cases}
$$

as desired.

Q.E.D.

4.2. Differential equations for $\boldsymbol{F}_{\mu, d^{l}}^{\rho}$. Since $\boldsymbol{F}_{\mu, d}^{\rho}\left(y e^{i \theta}\right)$ is even, it would be convenient to set $4 u=y^{2} e^{2 i \theta}$ and consider $\boldsymbol{F}_{\mu, d}^{\rho}(2 \sqrt{u})$. Assume that $|\operatorname{Re} \mu|<\operatorname{Re} \rho<\frac{1}{2}$ so that the integral $\boldsymbol{F}_{\mu, d}^{\rho}(2 \sqrt{u})$ is always convergent and gives rise to a continuous function. We are now going to verify

$$
\nabla_{\rho, \mu+d}\left(\boldsymbol{F}_{\mu, d}^{\rho}(2 \sqrt{u})\right)=0, \quad \bar{\nabla}_{\rho, \mu-d}\left(\boldsymbol{F}_{\mu, d}^{\rho}(2 \sqrt{u})\right)=0,
$$

with the hypergeometric differential operators $\nabla_{\rho, \mu+d}$ and $\bar{\nabla}_{\rho, \mu-d}$ defined as in (3.2) and (3.3) respectively. By symmetry, we only need to verify the former, which, if we set $v=\mu+d$ for simplify, may be explicitly written as

$$
4 u(1-u) \frac{\partial^{2} \boldsymbol{F}_{\mu, d}^{\rho}(2 \sqrt{u})}{\partial u^{2}}+2(1-2(\rho+1) u) \frac{\partial \boldsymbol{F}_{\mu, d}^{\rho}(2 \sqrt{u})}{\partial u}-\left(\rho^{2}-v^{2}\right) \boldsymbol{F}_{\mu, d}^{\rho}(2 \sqrt{u})=0 .
$$

For $s, r=0,1,2$, with $s+r=0,1,2$, we introduce

$$
\boldsymbol{I}_{s, r, \mu, d}^{\rho}(u)=\frac{1}{2 \sqrt{u^{s}}} \iint_{\mathbb{C} \backslash\{0\}} z^{\rho+s+r-1} \bar{z}^{\rho-1}(\partial / \partial z)^{r} \boldsymbol{J}_{\mu, d}(z) e(-2(z \sqrt{u}+\bar{z} \sqrt{\bar{u}})) i d z \wedge d \bar{z} .
$$

The integral $\boldsymbol{I}_{s, r, \mu, d}^{\rho}(u)$ should be regarded as distribution on $\mathbb{C}$. Precisely, for any Schwartz function $f(u) \in \mathcal{S}(\mathbb{C})$ (the Schwartz space on $\mathbb{C}$ is denoted by $S(\mathbb{C})$ as usual), let

$$
\left\langle u^{t} \boldsymbol{I}_{s, r, \mu, d}^{\rho}, f\right\rangle=\iint_{\mathbb{C} \backslash\{0\}} z^{\rho+s+r-1} \bar{z}^{\rho-1}(\partial / \partial z)^{r} \boldsymbol{J}_{\mu, d}(z) f^{\sharp}(z) i d z \wedge d \bar{z},
$$

with $f^{\sharp}(z) \in \mathcal{S}(\mathbb{C})$ given by

$$
f^{\sharp}(z)=\iint_{\mathbb{C} \backslash\{0\}} u^{1+2 t-s} \bar{u} f\left(u^{2}\right) e(-2(z u+\overline{z u})) i d u \wedge d \bar{u},
$$

in which $t=0,1,2$ or $t=-1$ only if $s=0,1$. Note here that $f^{\sharp}(z / 2)$ is the Fourier transform of $u^{1+2 t-s} \bar{u} f\left(u^{2}\right)$ and hence is also Schwartz. In the theory of distributions, we are free to differentiate under the integral and integrate by parts in the formal manner as what we shall do in the following.

To start with, note that $\boldsymbol{F}_{\mu, d}^{\rho}(2 \sqrt{u})=\boldsymbol{I}_{0,0, \mu, d}^{\rho}(u)$. For brevity, we put $\boldsymbol{I}_{s, r}=\boldsymbol{I}_{s, r, \mu, d}^{\rho}$ and $\boldsymbol{I}_{s}=\boldsymbol{I}_{s, 0}$.

First, for $s=0,1$, we have

$$
\frac{\partial \boldsymbol{I}_{s}}{\partial u}=-\frac{s}{2 u} \boldsymbol{I}_{s}-2 \pi i \boldsymbol{I}_{s+1}
$$

and

$$
\frac{\partial^{2} \boldsymbol{I}_{0}}{\partial u^{2}}=-2 \pi i \frac{\partial \boldsymbol{I}_{1}}{\partial u}=\frac{\pi i}{u} \boldsymbol{I}_{1}-4 \pi^{2} \boldsymbol{I}_{2}
$$

Second, for $s, r=0,1$, with $s+r=0,1$, by partial integration,

$$
4 \pi i \sqrt{u} \iint_{\mathbb{C} \backslash\{0\}} z^{\rho+s+r} \bar{z}^{\rho-1}(\partial / \partial z)^{r} \boldsymbol{J}_{\mu, d}(z) e(-2(z \sqrt{u}+\bar{z} \sqrt{\bar{u}})) i d z \wedge d \bar{z}
$$




$$
\begin{aligned}
=(\rho+s+ & r) \iint_{\mathbb{C} \backslash\{0\}} z^{\rho+s+r-1} \bar{z}^{\rho-1}(\partial / \partial z)^{r} \boldsymbol{J}_{\mu, d}(z) e(-2(z \sqrt{u}+\bar{z} \sqrt{\bar{u}})) i d z \wedge d \bar{z} \\
& +\iint_{\mathbb{C} \backslash\{0\}} z^{\rho+s+r} \bar{z}^{\rho-1}(\partial / \partial z)^{r+1} \boldsymbol{J}_{\mu, d}(z) e(-2(z \sqrt{u}+\bar{z} \sqrt{\bar{u}})) i d z \wedge d \bar{z} .
\end{aligned}
$$

It follows that

$$
4 \pi i u \boldsymbol{I}_{s+1, r}=(\rho+s+r) \boldsymbol{I}_{s, r}+\boldsymbol{I}_{s, r+1},
$$

and hence

$$
-16 \pi^{2} u^{2} \boldsymbol{I}_{2}=4 \pi i(\rho+1) u \boldsymbol{I}_{1}+4 \pi i u \boldsymbol{I}_{1,1}=\left(\rho^{2}+\rho\right) \boldsymbol{I}_{0}+(2 \rho+2) \boldsymbol{I}_{0,1}+\boldsymbol{I}_{0,2} .
$$

Third, since $\nabla_{\mu+d}\left(\boldsymbol{J}_{\mu, d}(z / 4 \pi)\right)=0$ (see (2.11) and (2.13)), we have

$$
\boldsymbol{I}_{0,2}+\boldsymbol{I}_{0,1}+16 \pi^{2} u \boldsymbol{I}_{2,0}-v^{2} \boldsymbol{I}_{0,0}=0 \quad(v=\mu+d) .
$$

Finally, combining these, we have

$$
\begin{aligned}
& 4 u(1-u) \frac{\partial^{2} \boldsymbol{I}_{0}}{\partial u^{2}}+2(1-2(\rho+1) u) \frac{\partial \boldsymbol{I}_{0}}{\partial u}-\left(\rho^{2}-v^{2}\right) \boldsymbol{I}_{0} \\
= & 4 u(1-u)\left(\frac{\pi i}{u} \boldsymbol{I}_{1}-4 \pi^{2} \boldsymbol{I}_{2}\right)-4 \pi i(1-2(\rho+1) u) \boldsymbol{I}_{1}-\left(\rho^{2}-v^{2}\right) \boldsymbol{I}_{0} \\
= & -16 \pi^{2} u \boldsymbol{I}_{2}+16 \pi^{2} u^{2} \boldsymbol{I}_{2}+4 \pi i(2 \rho+1) u \boldsymbol{I}_{1}-\left(\rho^{2}-v^{2}\right) \boldsymbol{I}_{0} \\
= & -16 \pi^{2} u \boldsymbol{I}_{2}-\left(\left(\rho^{2}+\rho\right) \boldsymbol{I}_{0}+(2 \rho+2) \boldsymbol{I}_{0,1}+\boldsymbol{I}_{0,2}\right)+(2 \rho+1)\left(\rho \boldsymbol{I}_{0}+\boldsymbol{I}_{0,1}\right)-\left(\rho^{2}-v^{2}\right) \boldsymbol{I}_{0} \\
= & v^{2} \boldsymbol{I}_{0}-16 \pi^{2} u \boldsymbol{I}_{2}-\boldsymbol{I}_{0,1}-\boldsymbol{I}_{0,2} \\
= & 0,
\end{aligned}
$$

as desired.

4.3. Conclusion. Combining (4.3) and (4.6), we deduce from Lemma 3.1 that, under the conditions $|\operatorname{Re} \mu|<\operatorname{Re} \rho<\frac{1}{2}$ and $\mu \neq 0$,

$$
\boldsymbol{F}_{\mu, d}^{\rho}(2 \sqrt{u})=4^{-\mu-\rho} C_{\mu, d}^{\rho} \cdot G_{\rho, \mu+d}^{(1)}(u) G_{\rho, \mu-d}^{(1)}(\bar{u})+4^{\mu-\rho} C_{-\mu,-d}^{\rho} \cdot G_{\rho, \mu+d}^{(2)}(u) G_{\rho, \mu-d}^{(2)}(\bar{u}),
$$

for all $u$ in the complex plane (the choice of the square root $\sqrt{u}$ is not essential since $\boldsymbol{F}_{\mu, d}^{\rho}$ is even). Let $2 \sqrt{u}=y e^{i \theta}$. In view of the formulae for $G_{\rho, v}^{(1)}$ and $G_{\rho, v}^{(2)}$ in (3.5) and the definition of $\boldsymbol{F}_{\mu, d}^{\rho}$ in (4.1), it is clear that (4.7) is equivalent to (1.22) in Theorem 1.1 Finally, thanks to the principal of analytic continuation, it follows from Lemma 4.1 (1) that the condition $\mu \neq 0$ may be removed and the condition $|\operatorname{Re} \mu|<\operatorname{Re} \rho<\frac{1}{2}$ may be improved into $|\operatorname{Re} \mu|<\operatorname{Re} \rho<1$ if $y>2$. Note that in the definition of $C_{\mu, d}^{\rho}$ the zero of $\sin (\pi \mu)$ is annihilated by the pole of $\Gamma(1+\mu+d) \Gamma(1+\mu-d)$ at $\mu=0$ if $d \neq 0$.

\section{Proof of Theorem 1.4}

Consider

$$
\boldsymbol{F}_{\mu, d}^{\rho}\left(y e^{i \theta}\right)-\boldsymbol{P}_{\mu, d}^{\rho}\left(y e^{i \theta}\right)=\int_{0}^{2 \pi} \int_{0}^{\infty} \boldsymbol{M}_{\mu, d}\left(x e^{i \phi}\right) e(-2 x y \cos (\phi+\theta)) x^{2 \rho-1} d x d \phi,
$$

in which $\boldsymbol{M}_{\mu, d}(z)=\boldsymbol{J}_{\mu, d}(z)-\boldsymbol{R}_{\mu, d}(z)$ is the regularized Bessel function defined in (1.26). It is examined in Lemma 4.1 that for the convergence of this integral at infinity we need $\operatorname{Re} \rho<\frac{1}{2}-|\operatorname{Re} \mu|$ and also $y>0$. On the other hand, in view of (2.16), the convergence at zero is secured if $|\operatorname{Re} \mu|-1<\operatorname{Re} \rho$. 
LeMma 5.1. The integral $\boldsymbol{F}_{\mu, d}^{\rho}\left(y e^{i \theta}\right)-\boldsymbol{P}_{\mu, d}^{\rho}\left(y e^{i \theta}\right)$ is convergent for $y>0$ if $|\operatorname{Re} \mu|-1<$ $\operatorname{Re} \rho<\frac{1}{2}-|\operatorname{Re} \mu|$, uniformly for $\operatorname{Re} \mu$ and $\operatorname{Re} \rho$ in compact sets.

Let $C_{\mu, d}^{\rho}$ and $F_{\rho, v}^{(1,2)}(z)$ be defined in 1.20) and (1.21). By Theorem 1.1 and Lemma 4.3. we have

$$
\begin{array}{r}
\boldsymbol{F}_{\mu, d}^{\rho}\left(y e^{i \theta}\right)-\boldsymbol{P}_{\mu, d}^{\rho}\left(y e^{i \theta}\right)=\frac{C_{\mu, d}^{\rho}}{y^{2 \mu+2 \rho} e^{2 i d \theta}}\left(F_{\rho, \mu+d}^{(1)}\left(4 / y^{2} e^{2 i \theta}\right) F_{\rho, \mu-d}^{(1)}\left(4 e^{2 i \theta} / y^{2}\right)-1\right) \\
+C_{-\mu,-d}^{\rho} y^{2 \mu-2 \rho} e^{2 i d \theta}\left(F_{\rho, \mu+d}^{(2)}\left(4 / y^{2} e^{2 i \theta}\right) F_{\rho, \mu-d}^{(2)}\left(4 e^{2 i \theta} / y^{2}\right)-1\right) .
\end{array}
$$

provided that $|\operatorname{Re} \mu|<\operatorname{Re} \rho<\frac{1}{2}-|\operatorname{Re} \mu|$. We claim that (5.1) remains valid in the extended range $|\operatorname{Re} \mu|-1<\operatorname{Re} \rho<\frac{1}{2}-|\operatorname{Re} \mu|$. For $d \neq 0$, the validity of (5.1) extends to $|\operatorname{Re} \mu|-1<\operatorname{Re} \rho<\frac{1}{2}-|\operatorname{Re} \mu|$, since $C_{\mu, d}^{\rho}$ is analytic in this range. Now consider the case $d=0$. For simplicity, let us assume $\mu \neq 0$. Then $C_{\mu, 0}^{\rho}$ has a simple pole at $\rho=-\mu$. However, we have $F_{\rho, \mu}^{(1)}(z)-1 \equiv 0$ if $\rho=-\mu$ and similarly $F_{\rho, \mu}^{(2)}(z)-1 \equiv 0$ if $\rho=\mu$. So the extension of 5.1 to the range $|\operatorname{Re} \mu|-1<\operatorname{Re} \rho<\frac{1}{2}-|\operatorname{Re} \mu|$ is still permissible.

Assume that $|\operatorname{Re} \mu|<\frac{1}{2}$. Let $\rho \rightarrow 0$. Since

$$
C_{\mu, d}^{0}=1 /\left(d^{2}-\mu^{2}\right)
$$

and

$$
\begin{aligned}
& F_{0, v}^{(1)}\left(z^{2}\right)={ }_{2} F_{1}\left(\frac{v}{2} ; \frac{1+v}{2} ; 1+v ; z^{2}\right)=\left(\frac{2}{1+\sqrt{1-z^{2}}}\right)^{v}, \\
& F_{0, v}^{(2)}\left(z^{2}\right)={ }_{2} F_{1}\left(-\frac{v}{2} ; \frac{1-v}{2} ; 1-v ; z^{2}\right)=\left(\frac{1+\sqrt{1-z^{2}}}{2}\right)^{v},
\end{aligned}
$$

we find that the limiting form of (5.1) as $\rho \rightarrow 0$ is exactly (1.28) in Theorem 1.4

\section{Proof of Theorem 1.6 over complex numbers}

To start with, we translate the formula 1.33 into our language for $F_{\infty}=\mathbb{C}$. According to the notation of Venkatesh, set $\phi(z)=\varphi\left(z^{2}\right)$ and $\sqrt{\kappa}=\left(k-\sqrt{k^{2}-4}\right) / 2(|k|>2)$, then the identity we need to prove is the following,

$$
\iint_{\mathbb{C} \backslash\{0\}} \phi(z) e(\operatorname{Tr}(k z)) \frac{i d z \wedge d \bar{z}}{|z|^{2}}=2 \int_{-\infty}^{\infty} h(t) \sinh (\pi t)\left|\frac{k+\sqrt{k^{2}-4}}{2}\right|^{-2 i t} \frac{d t}{t},
$$

where

$$
\phi(z)=\frac{1}{2} \int_{-\infty}^{\infty} h(t) \boldsymbol{J}_{i t}(z) \sinh (\pi t) t d t
$$

Recall here that $h(s)$ is a holomorphic even function in the strip $|\operatorname{Im} s| \leqslant M$, satisfying decay estimates

$$
h(t+i \sigma) \ll e^{-\pi|t|}(|t|+1)^{-N}
$$

Define $\omega(z)$ to be

$$
\omega(z)=\frac{1}{2} \int_{-\infty}^{\infty} h(t) \boldsymbol{R}_{i t}(z) \sinh (\pi t) t d t=i \int_{-\infty}^{\infty} h(t)|2 \pi z|^{2 i t} \frac{t d t}{\Gamma(1+i t)^{2}},
$$


where $\boldsymbol{R}_{i t}(z)=\boldsymbol{R}_{i t, 0}(z)$ is defined as in $\left.1.24,1.25\right)$. Note that $\omega(z)$ is simply the (horizontal) Mellin inverse transform of the function $i(2 \pi)^{2 i t} h(t) t / \Gamma(1+i t)^{2}$.

We quote from Lemma 4.1 in [Qi4] the following uniform estimate for $\boldsymbol{J}_{i t}(z)$,

$$
t \boldsymbol{J}_{i t}(z) \ll(|t|+1)^{3} \min \{1,1 /|z|\} .
$$

Further, it is clear from [Wat $3.13(1)]$ (note that $|\Gamma(1+i t)|^{2}=\pi t / \sinh (\pi t)$ ) that

$$
t\left(\boldsymbol{J}_{i t}(z)-\boldsymbol{R}_{i t}(z)\right) \ll|z|^{2}, \quad|z| \leqslant 1
$$

We first write the integral on the left of (6.1) as follows,

$$
\iint_{\mathbb{C} \backslash\{0\}}(\phi(z)-\omega(z)) e(\operatorname{Tr}(k z)) \frac{i d z \wedge d \bar{z}}{|z|^{2}}+\iint_{\mathbb{C} \backslash\{0\}} \omega(z) e(\operatorname{Tr}(k z)) \frac{i d z \wedge d \bar{z}}{|z|^{2}}
$$

Applying (6.2) and (6.4), the first integral in (6.7) is equal to

$$
\frac{1}{2} \iint_{\mathbb{C} \backslash\{0\}}\left(\int_{-\infty}^{\infty} h(t)\left(\boldsymbol{J}_{i t}(z)-\boldsymbol{R}_{i t}(z)\right) \sinh (\pi t) t d t\right) e(\operatorname{Tr}(k z)) \frac{i d z \wedge d \bar{z}}{|z|^{2}} .
$$

Making crucial use of (6.3), (6.5) and (6.6), we verify that the double integral is absolutely convergent except for the contribution from $\boldsymbol{R}_{i t}(z)$ in the vicinity of $z=\infty$. Nevertheless, we may switch the order of integration. This is because the integral becomes absolutely convergent after integration by part in a neighborhood of $z=\infty$ (see Lemma2.1); we still need (6.3) to secure this. We then obtain

$$
\frac{1}{2} \int_{-\infty}^{\infty} h(t)\left(\iint_{\mathbb{C} \backslash\{0\}}\left(\boldsymbol{J}_{i t}(z)-\boldsymbol{R}_{i t}(z)\right) e(\operatorname{Tr}(k z)) \frac{i d z \wedge d \bar{z}}{|z|^{2}}\right) \sinh (\pi t) t d t .
$$

The inner integral is evaluated in Theorem 1.4 . Thus

$$
\begin{aligned}
\iint_{\mathbb{C} \backslash\{0\}}(\phi(z)-\omega(z)) e(\operatorname{Tr}(k z)) \frac{i d z \wedge d \bar{z}}{|z|^{2}}= \\
2 \int_{-\infty}^{\infty} h(t)\left(\left|\left(k+\sqrt{k^{2}-4}\right) / 2\right|^{-2 i t}-|k|^{-2 i t}\right) \sinh (\pi t) \frac{d t}{t} .
\end{aligned}
$$

Now consider the second integral in 6.7). Applying (6.4), we have

$$
\iint_{\mathbb{C} \backslash\{0\}} \omega(z) e(\operatorname{Tr}(k z)) \frac{i d z \wedge d \bar{z}}{|z|^{2}}=i \iint_{\mathbb{C} \backslash\{0\}}\left(\int_{-\infty}^{\infty} h(t)|2 \pi z|^{2 i t} \frac{t d t}{\Gamma(1+i t)^{2}}\right) e(\operatorname{Tr}(k z)) \frac{i d z \wedge d \bar{z}}{|z|^{2}} .
$$

Let $\sigma>0$ be small, say $\sigma<\frac{1}{2}$. In view of $(6.3$, we may shift the line of integration in the inner integral to $\operatorname{Im} t=-\sigma$, then the integral on the right turns into

$$
i \iint_{\mathbb{C} \backslash\{0\}}\left(\int_{-i \sigma-\infty}^{-i \sigma+\infty} h(t)|2 \pi z|^{2 i t} \frac{t d t}{\Gamma(1+i t)^{2}}\right) e(\operatorname{Tr}(k z)) \frac{i d z \wedge d \bar{z}}{|z|^{2}} .
$$

Again, we may switch the order of integration, although this integral is not absolutely convergent in the vicinity of $z=\infty$, obtaining

$$
i \int_{-i \sigma-\infty}^{-i \sigma+\infty} h(t)\left(\iint_{\mathbb{C} \backslash\{0\}}|2 \pi z|^{2 i t} e(\operatorname{Tr}(k z)) \frac{i d z \wedge d \bar{z}}{|z|^{2}}\right) \frac{t d t}{\Gamma(1+i t)^{2}} .
$$

The inner integral is evaluated in Lemma4.4(it is convergent actually as a double integral, since $\left.0<\operatorname{Re}(i t)=\sigma<\frac{1}{2}\right)$. Thus we have

$$
\iint_{\mathbb{C} \backslash\{0\}} \omega(z) e(\operatorname{Tr}(k z)) \frac{i d z \wedge d \bar{z}}{|z|^{2}}=2 \int_{-i \sigma-\infty}^{-i \sigma+\infty} h(t)|k|^{-2 i t} \sinh (\pi t) \frac{d t}{t}
$$


Utilizing again $(6.3)$, we are now free to move the line of integration back to $\operatorname{Im} t=0$, obtaining

$$
\iint_{\mathbb{C} \backslash\{0\}} \omega(z) e(\operatorname{Tr}(k z)) \frac{i d z \wedge d \bar{z}}{|z|^{2}}=2 \int_{-\infty}^{\infty} h(t)|k|^{-2 i t} \sinh (\pi t) \frac{d t}{t} .
$$

In conclusion, the formula (6.1) is proven by summing (6.8) and (6.9).

\section{Appendix A.}

In this appendix, we record some extensions of the general Weber-Schafheitlin formula in Theorem 1.1. These results may be proven by modifying our arguments in $\S \S 3$ and4.

Let $\mu$ be a complex number and $d$ be an integer or half-integer. Define

$$
J_{\mu, d}(z)=J_{\mu+d}(z) J_{\mu-d}(\bar{z}) .
$$

and

$$
\boldsymbol{J}_{\mu, d}(z)=\left\{\begin{array}{l}
\frac{1}{\sin (\pi \mu)}\left(J_{\mu, d}(4 \pi z)-J_{-\mu,-d}(4 \pi z)\right), \text { if } 2 d \text { is even, } \\
\frac{i}{\cos (\pi \mu)}\left(J_{\mu, d}(4 \pi z)+J_{-\mu,-d}(4 \pi z)\right), \text { if } 2 d \text { is odd. }
\end{array}\right.
$$

We need to take the limit in the non-generic case when $\mu-d$ is an integer. Note that $\boldsymbol{J}_{\mu, d}(z)$ is an even or odd function according as $2 d$ is even or odd.

Let $\rho$ be a complex number and $m$ be an integer. We consider the following integrals

$$
\begin{aligned}
& \boldsymbol{F}_{\mu, d}^{\rho, m}\left(y e^{i \theta}\right)=\int_{0}^{2 \pi} \int_{0}^{\infty} \boldsymbol{J}_{\mu, d}\left(x e^{i \phi}\right) \cos (4 \pi x y \cos (\phi+\theta)) x^{2 \rho-1} e^{i m \phi} d x d \phi, \\
& \boldsymbol{G}_{\mu, d}^{\rho, m}\left(y e^{i \theta}\right)=\int_{0}^{2 \pi} \int_{0}^{\infty} \boldsymbol{J}_{\mu, d}\left(x e^{i \phi}\right) \sin (4 \pi x y \cos (\phi+\theta)) x^{2 \rho-1} e^{i m \phi} d x d \phi .
\end{aligned}
$$

By simple parity considerations, it is clear that the former or the latter integral is zero according as $m+2 d$ is odd or even (as the integrand is an odd function).

We have the following generalization of Theorem 1.1 .

Theorem A.1. Let notation be as above. Define

$$
F_{v}^{\lambda}(z)={ }_{2} F_{1}\left(\frac{\lambda+v}{2} ; \frac{1+\lambda+v}{2} ; 1+v ; z\right) .
$$

Set

$$
\begin{aligned}
& C_{\mu, d}^{\rho, m}=-\frac{\Gamma(\rho+\mu+m / 2+d) \Gamma(\rho+\mu-m / 2-d)}{\Gamma(1+\mu+d) \Gamma(1+\mu-d)} \cdot\left\{\begin{array}{l}
\frac{\sin (\pi(\rho+\mu))}{(2 \pi)^{2 \rho} \sin (\pi \mu)}, \text { if } 2 d \text { is even } \\
\frac{i \sin (\pi(\rho+\mu))}{(2 \pi)^{2 \rho} \cos (\pi \mu)}, \text { if } 2 d \text { is odd }
\end{array}\right. \\
& D_{\mu, d}^{\rho, m}=-\frac{\Gamma(\rho+\mu+m / 2+d) \Gamma(\rho+\mu-m / 2-d)}{\Gamma(1+\mu+d) \Gamma(1+\mu-d)} \cdot\left\{\begin{array}{l}
\frac{\cos (\pi(\rho+\mu))}{(2 \pi)^{2 \rho} \sin (\pi \mu)}, \text { if } 2 d \text { is even, } \\
\frac{i \cos (\pi(\rho+\mu))}{(2 \pi)^{2 \rho} \cos (\pi \mu)}, \text { if } 2 d \text { is odd }
\end{array}\right.
\end{aligned}
$$


For all complex $z$, we have

$$
\boldsymbol{F}_{\mu, d}^{\rho, m}(z)=\frac{C_{\mu, d}^{\rho, m} F_{\mu+d}^{\rho+\frac{1}{2} m}\left(4 / z^{2}\right) F_{\mu-d}^{\rho-\frac{1}{2} m}\left(4 / \bar{z}^{2}\right)}{|z|^{2 \rho+2 \mu}(z /|z|)^{m+2 d}}+\frac{C_{-\mu,-d}^{\rho, m} F_{-\mu-d}^{\rho+\frac{1}{2} m}\left(4 / z^{2}\right) F_{-\mu+d}^{\rho-\frac{1}{2} m}\left(4 / \bar{z}^{2}\right)}{|z|^{2 \rho-2 \mu}(z /|z|)^{m-2 d}},
$$

if $m+2 d$ is even, and

$$
\boldsymbol{G}_{\mu, d}^{\rho, m}(z)=\frac{D_{\mu, d}^{\rho, m} F_{\mu+d}^{\rho+\frac{1}{2} m}\left(4 / z^{2}\right) F_{\mu-d}^{\rho-\frac{1}{2} m}\left(4 / \bar{z}^{2}\right)}{|z|^{2 \rho+2 \mu}(z /|z|)^{m+2 d}}+\frac{D_{-\mu,-d}^{\rho, m} F_{-\mu-d}^{\rho+\frac{1}{2} m}\left(4 / z^{2}\right) F_{-\mu+d}^{\rho-\frac{1}{2} m}\left(4 / \bar{z}^{2}\right)}{|z|^{2 \rho-2 \mu}(z /|z|)^{m-2 d}},
$$

if $m+2 d$ is odd, of which the former is valid when $|\operatorname{Re} \mu|<\operatorname{Re} \rho<\frac{1}{2}$ and the latter when $|\operatorname{Re} \mu|-\frac{1}{2}<\operatorname{Re} \rho<\frac{1}{2}$ (we need to take the limit in the identities when either $\mu=d=0$ or $\mu=|d|=\frac{1}{2}$ ). Moreover, for $|z|>2$, the former identity is valid when $|\operatorname{Re} \mu|<\operatorname{Re} \rho<1$ and the latter when $|\operatorname{Re} \mu|-\frac{1}{2}<\operatorname{Re} \rho<1$.

Corollary A.2. Set

$$
\begin{aligned}
& \Delta_{\mu, d}^{\rho}=\cos (\pi \rho) \sin (\pi(\rho+\mu)) \sin (\pi(\rho-\mu)) \cdot \begin{cases}1, & \text { if } 2 d \text { is even, } \\
i \tan (\pi \mu), & \text { if } 2 d \text { is odd },\end{cases} \\
& \Lambda_{\mu, d}^{\rho}=\cos (\pi \rho) \cos (\pi(\rho+\mu)) \cos (\pi(\rho-\mu)) \cdot \begin{cases}\cot (\pi \mu), & \text { if } 2 d \text { is even, } \\
i, & \text { if } 2 d \text { is odd },\end{cases}
\end{aligned}
$$

and

$$
\Gamma_{v}^{\lambda}=\Gamma(1 / 2-\lambda) \Gamma(\lambda+v) \Gamma(\lambda-v)
$$

We have

$$
\boldsymbol{F}_{\mu, d}^{\rho, m}(1)=\left(2 / \pi^{3}\right)(8 \pi)^{-2 \rho} \Delta_{\mu, d}^{\rho} \Gamma_{\mu+d}^{\rho+\frac{1}{2} m} \Gamma_{\mu-d}^{\rho-\frac{1}{2} m}
$$

for $|\operatorname{Re} \mu|<\operatorname{Re} \rho<\frac{1}{2}$ and $m+2 d$ even, and

$$
\boldsymbol{G}_{\mu, d}^{\rho, m}(1)=\left(2 / \pi^{3}\right)(8 \pi)^{-2 \rho} \Lambda_{\mu, d}^{\rho} \Gamma_{\mu+d}^{\rho+\frac{1}{2} m} \Gamma_{\mu-d}^{\rho-\frac{1}{2} m}
$$

for $|\operatorname{Re} \mu|-\frac{1}{2}<\operatorname{Re} \rho<\frac{1}{2}$ and $m+2 d$ odd.

In particular, for $|\operatorname{Re} \mu|<\operatorname{Re} \rho<\frac{1}{2}$ and half-integer $d$, we have

$$
\begin{aligned}
\int_{0}^{2 \pi} \int_{0}^{\infty} \boldsymbol{J}_{\mu, d}\left(x e^{i \phi}\right) e(-4 x \cos \phi) x^{2 \rho-1} d x d \phi=\left(2 / \pi^{3}\right)(8 \pi)^{-2 \rho} \cos (\pi \rho) \Gamma(1 / 2-\rho)^{2} . \\
\\
\cos (\pi(\rho+\mu)) \cos (\pi(\rho-\mu)) \Gamma(\rho+\mu+d) \Gamma(\rho+\mu-d) \Gamma(\rho-\mu+d) \Gamma(\rho-\mu-d),
\end{aligned}
$$

which is an analogue of the formula in Corollary 1.3

\section{References}

[Alt1] S. A. Altuğ. Beyond endoscopy via the trace formula: 1. Poisson summation and isolation of special representations. Compos. Math., 151(10):1791-1820, 2015.

[Alt2] S. A. Altuğ. Beyond endoscopy via the trace formula, II: Asymptotic expansions of Fourier transforms and bounds towards the Ramanujan conjecture. Amer. J. Math., 139(4):863-913, 2017.

[Alt3] S. A. Altuğ. Beyond endoscopy via the trace formula, III: The standard representation. J. Inst. Math. Jussieu, 2018. doi:10.1017/S1474748018000427.

[BM1] E. M. Baruch and Z. Mao. Bessel identities in the Waldspurger correspondence over a $p$-adic field. Amer. J. Math., 125(2):225-288, 2003.

[BM2] E. M. Baruch and Z. Mao. Bessel identities in the Waldspurger correspondence over the real numbers. Israel J. Math., 145:1-81, 2005. 
[BM3] E. M. Baruch and Z. Mao. Central value of automorphic L-functions. Geom. Funct. Anal., 17(2):333384, 2007.

[BM4] R. W. Bruggeman and R. J. Miatello. Sum formula for $\mathrm{SL}_{2}$ over a number field and Selberg type estimate for exceptional eigenvalues. Geom. Funct. Anal., 8(4):627-655, 1998.

[BM5] R. W. Bruggeman and Y. Motohashi. Sum formula for Kloosterman sums and fourth moment of the Dedekind zeta-function over the Gaussian number field. Funct. Approx. Comment. Math., 31:23-92, 2003.

[CQ1] J. Chai and Z. Qi. Bessel identities in the Waldspurger correspondence over the complex numbers. arXiv:1802.01229. to appear in Israel J. Math., 2018.

[CQ2] J. Chai and Z. Qi. On the Waldspurger formula and the metaplectic Ramanujan conjecture over number fields. J. Funct. Anal., 2019. doi:10.1016/j.jfa.2019.05.013.

[FLN] E. Frenkel, R. Langlands, and B. C. Ngô. Formule des traces et fonctorialité: le début d'un programme. Ann. Sci. Math. Québec, 34(2):199-243, 2010.

[GR] I. S. Gradshteyn and I. M. Ryzhik. Table of Integrals, Series, and Products. Elsevier/Academic Press, Amsterdam, Seventh edition, 2007.

[Her1] P. E. Herman. Beyond endoscopy for the Rankin-Selberg L-function. J. Number Theory, 131(9):16911722, 2011.

[Her2] P. E. Herman. The functional equation and beyond endoscopy. Pacific J. Math., 260(2):497-513, 2012.

[Her3] P. E. Herman. Quadratic base change and the analytic continuation of the Asai $L$-function: a new trace formula approach. Amer. J. Math., 138(6):1669-1729, 2016.

[Lan] R. P. Langlands. Beyond endoscopy. In Contributions to automorphic forms, geometry, and number theory, pages 611-697. Johns Hopkins Univ. Press, Baltimore, MD, 2004.

[LL] J.-P. Labesse and R. P. Langlands. L-indistinguishability for SL(2). Canad. J. Math., 31(4):726-785, 1979.

[MOS] W. Magnus, F. Oberhettinger, and R. P. Soni. Formulas and Theorems for the Special Functions of Mathematical Physics. Third enlarged edition. Die Grundlehren der mathematischen Wissenschaften, Band 52. Springer-Verlag New York, Inc., New York, 1966.

[MW] R. Miatello and N. R. Wallach. Kuznetsov formulas for real rank one groups. J. Funct. Anal., 93(1):171206, 1990.

[Olv] F. W. J. Olver. Asymptotics and Special Functions. Academic Press, New York-London, 1974. Computer Science and Applied Mathematics.

[Qi1] Z. Qi. Theory of fundamental Bessel functions of high rank. arXiv:1612.03553, to appear in Mem. Amer. Math. Soc., 2016.

[Qi2] Z. Qi. On the Fourier transform of Bessel functions over complex numbers-I: the spherical case. Monatsh. Math., 186(3):471-479, 2018.

[Qi3] Z. Qi. On the Fourier transform of Bessel functions over complex numbers-II: the general case. Trans. Amer. Math. Soc., 372:2829-2854, 2019.

[Qi4] Z. Qi. Subconvexity for twisted $L$-functions on $\mathrm{GL}_{3}$ over the Gaussian number field. Trans. Amer. Math. Soc., 2019. doi:10.1090/tran/7710.

[Sak1] Y. Sakellaridis. Beyond endoscopy for the relative trace formula I: Local theory. In Automorphic representations and L-functions, volume 22 of Tata Inst. Fundam. Res. Stud. Math., pages 521-590. Tata Inst. Fund. Res., Mumbai, 2013.

[Sak2] Y. Sakellaridis. Beyond endoscopy for the relative trace formula II: Global theory. J. Inst. Math. Jussieu, 18(2):347-447, 2019.

[Sar] P. Sarnak. Comments on Langlands' lecture "Endoscopy and Beyond". 2001. http://publications.ias.edu/sites/default/files/SarnakLectureNotes-1.pdf

[Ven1] A. Venkatesh. Limiting Forms of the Trace Formula. Ph.D. Thesis. Princeton University, 2002.

[Ven2] A. Venkatesh. "Beyond Endoscopy" and special forms on GL(2). J. Reine Angew. Math., 577:23-80, 2004.

[Wat] G. N. Watson. A Treatise on the Theory of Bessel Functions. Cambridge University Press, Cambridge, England; The Macmillan Company, New York, 1944. 
[Whi] P.-J. White. The base change $L$-function for modular forms and Beyond Endoscopy. J. Number Theory, 140:13-37, 2014.

School of Mathematical Sciences, Zhejiang University, Hangzhou, 310027, China

E-mail address: zhi.qi@zju.edu.cn 